\title{
Optimal tightening process of bolted joints
}

\author{
Jean-Michel Monville* \\ MZ Intelligent Systems, 21 Boulevard de Lozère, 91120 Palaiseau, France
}

Received 28 April 2016 / Accepted 17 June 2016

\begin{abstract}
Threaded fasteners were developed long time (let's remember that Archimedes - 287-212 BC - invented the water screw). Nowadays, bolted joints are used in almost all sectors of the industry. But in spite of having been an important machine part for centuries, problems may be encountered with them. They are so common that they are taken for granted and too often, not analyzed as deeply as it should be. The wrong tightening is one of the most frequent causes of ductile rupture and by far the most frequent cause of fatigue failure. The tightening operation is never easy. It is necessary to pay particular attention to the choice of the tightening tool, the process and the control method. The tightening operation may itself cause damage on parts. The tightening load must not be too low, or excessive or not equally distributed among the bolts. These three defects can even be made on the same bolted joint! This impacts badly the performance of the assembly and leads to a shorter lifespan. If insufficient precautions are taken, the real tightening preload on all the bolts will not fit well with the requirements and would be badly distributed. Consequently, the practical conditions are quite different from the hypothesizes which are taken for the initial calculations (analytics or FEM) at the design stage. Thus, the results of the calculations of bolted joints cannot be considered as accurate and reliable. Practically, there are several means to tighten a bolt. The two ways most frequently used are torque wrench and hydraulic bolt tensioner. Torque wrench involves exerting a torque to the bolt head or the nut. Hydraulic bolt tensioner applies a traction load directly on the bolt. It is well known that bolt tensioners give better accuracy and homogeneity in the final tightening load than the torque method, but the tension load applied with the tensioner must be higher than the final remaining tightening load. So, the paper focusses on the hydraulic tensioner to show how the tightening load can be obtained from the applied tension load and to propose a way to optimize and secure the tightening process. However, for the reasons above mentioned it appears necessary to first make a general description of technical aspects of bolted joints. What really happens when tightening with torque wrench or with bolt tensioner is explained.
\end{abstract}

Key words: Bolted joints, Multiple bolts, Tightening preload, Optimum tightening process.

\section{Part I: classical elements on bolted joints}

\subsection{Usual configurations of the bolted joints}

A bolt is composed of a screw or a stud with its nut(s).

We can have a screw directly screwed in a structure or a screw going through with a nut at opposite end of the head or a stud screwed in structure with one nut at the other end or a stud going through with one nut at each end.

Various types of loadings can be applied to the bolted joints: direct compression or traction loads, bending moment, internal pressure, shear loading, torsion, cyclic loading, vibrations, etc.

We find bolted joints in almost all industries such as: air and spacecraft, automobile, railway, nuclear, wind-turbine, petro-chemistry, civil engineering, shipping industry, defense, machine tool and so...

The range of size of bolts is extremely large, from less than $2 \mathrm{~mm}$ to more than $500 \mathrm{~mm}$.

*e-mail: jeanmichelmonville@gmail.com

\subsection{Tightening load (preload) (Refs. [1-3])}

The tightening load generated after the tightening operation is a tension load in the bolt and a compression load on the joint members to prevent any movement between them (Figures 1 and 2).

If a movement occurs between the joint members, it means that the bolted joint is failing. This is the most common cause of structural joint failure.

This is often due to the tightening operation not having been carried out properly.

\subsection{Application of an external load (Refs. [1-3])}

When an external traction load is applied to the assembly we have the following graph (Figures 3 and 4).

Only a part of the external load is applied to the bolt when the tightening load is sufficient.

Figure 5 illustrates an external compression load being applied to the assembly. 

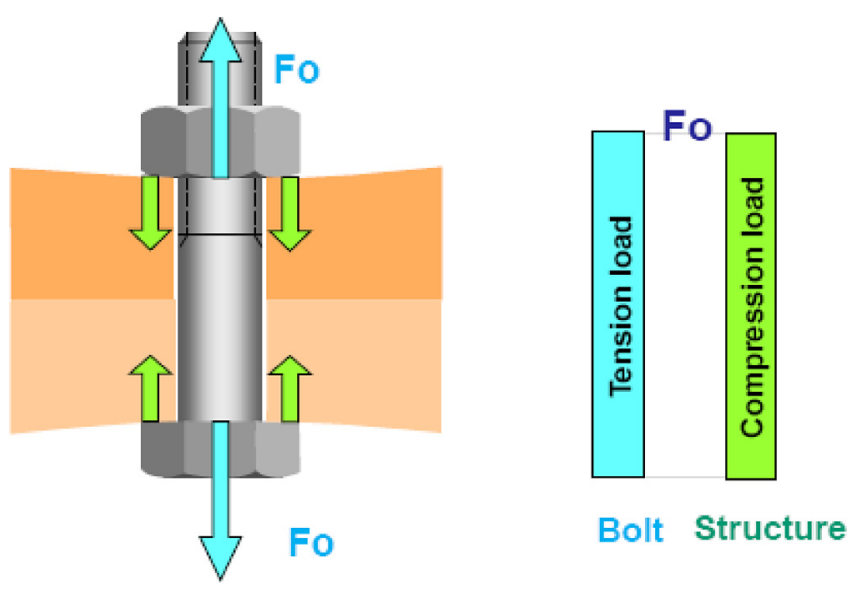

Figure 1. Schematic presentation of tightening load in a bolted joint.

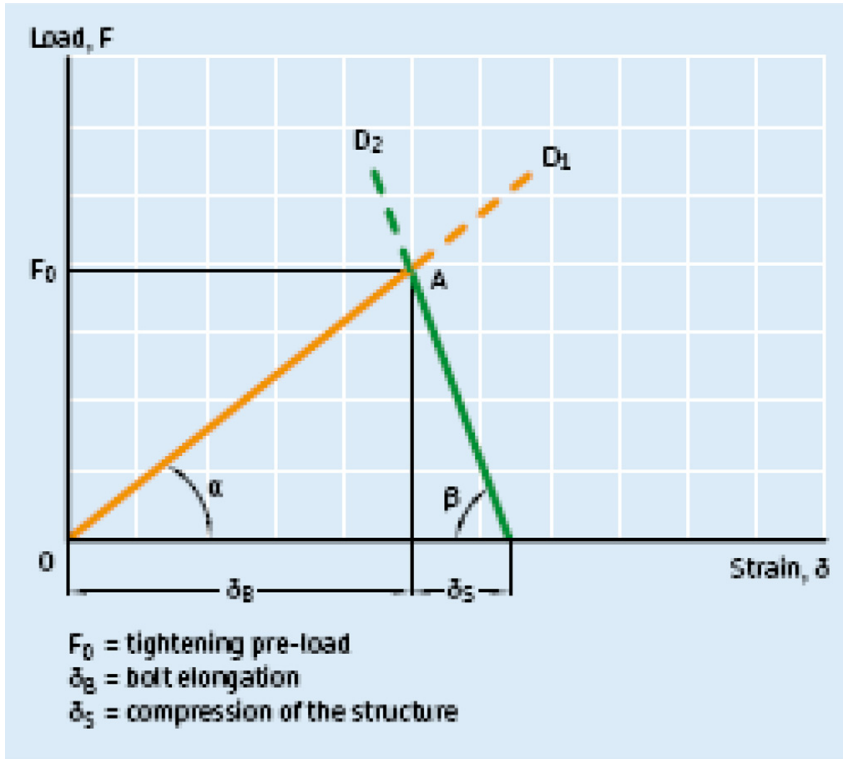

Figure 2. Graph of tightening load in a bolted joint. Tension of bolt is in yellow and compression of structure in green.
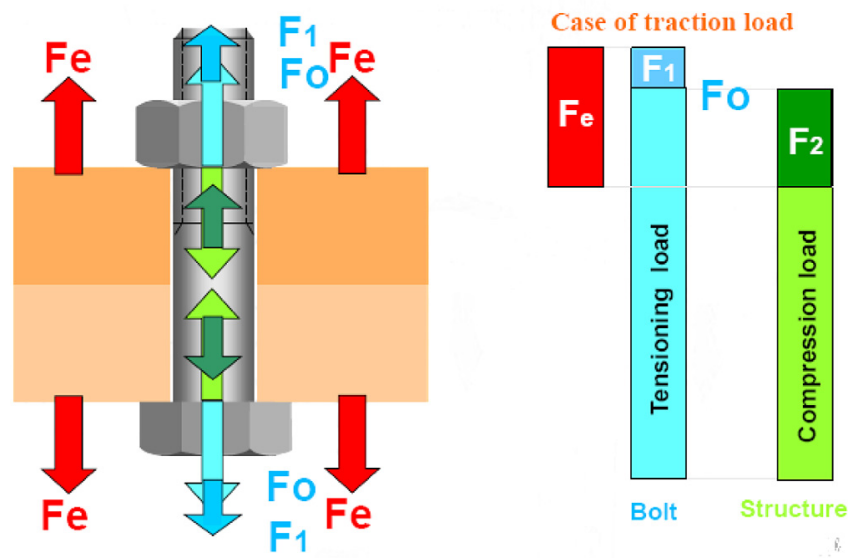

Figure 3. Schematic presentation of an external traction load on a tightened bolted joint.

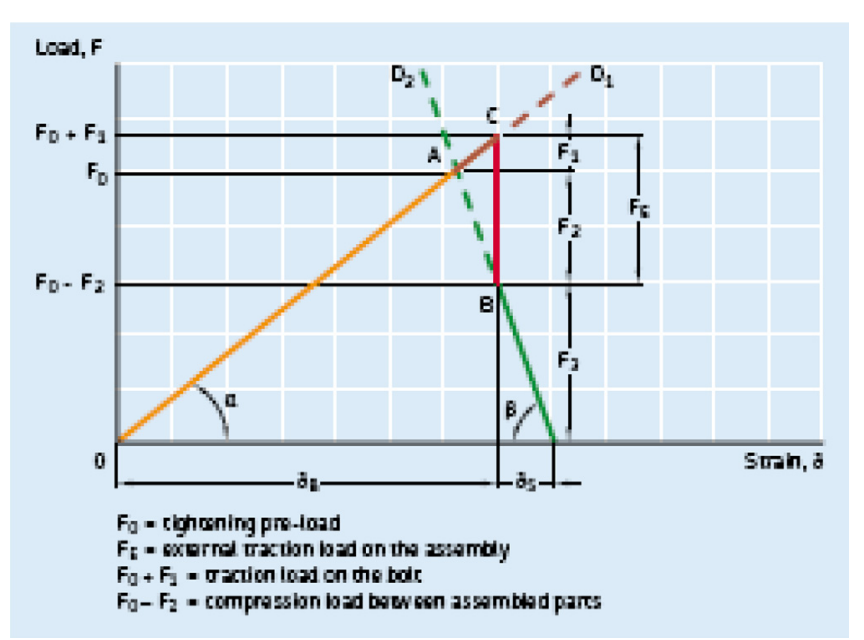

Figure 4. Graph of application of an external traction load on a tightened bolted joint.

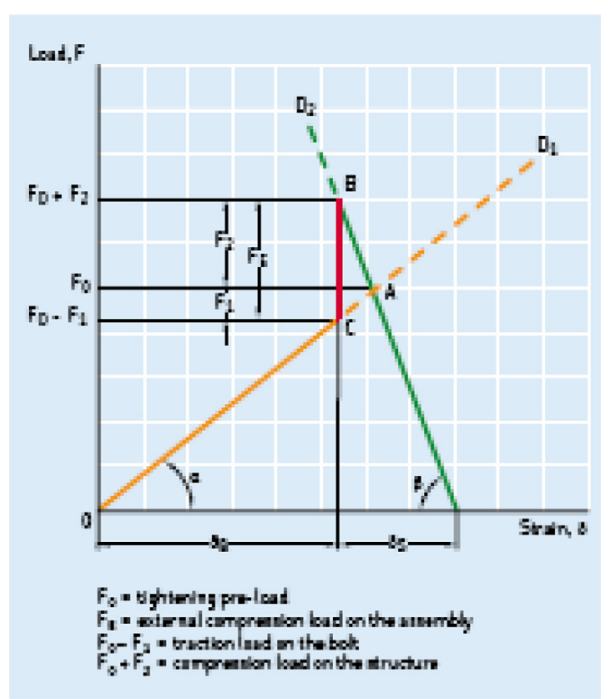

Figure 5. Graph of application of an external compression load on a tightened bolted joint.

However, as traction load is more common than compression load and generates higher stress levels, it is we will analyse in this document.

The loads on parts depend on the stiffness of each part, bolt $K_{\mathrm{b}}$ and structure $K_{\mathrm{s}}$.

For the traction load on bolt we come to:

$$
F_{\mathrm{b}}=F_{\mathrm{o}}+F_{\mathrm{e}} \frac{K_{\mathrm{b}}}{K_{\mathrm{b}}+K_{\mathrm{s}}} .
$$

For the residual compression load on the structure we have:

$$
F_{\mathrm{s}}=F_{\mathrm{o}}-F_{\mathrm{e}} \frac{K_{\mathrm{s}}}{K_{\mathrm{b}}+K_{\mathrm{s}}} .
$$

More accurately, the load on bolt and the residual compression on the structure depend on the way the external load is applied (Figure 6). 


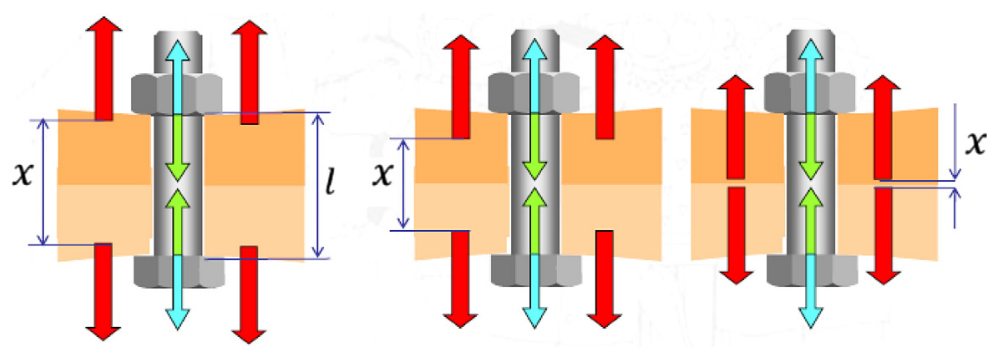

Figure 6. Schematic presentation of an external traction load on a tightened bolted joint at different points.

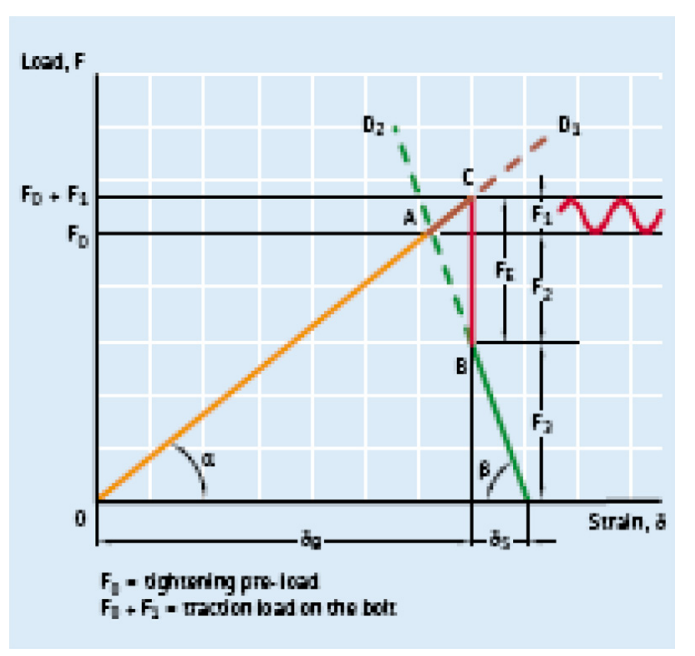

Figure 7. Graph of cyclic loading of a tightened bolted joint.

Two factors are defined to take this into account (see Ref. [1]). They are related to the distance $x$ shown on the following picture.

Load application coefficient: $\gamma=\frac{x}{l}$. Load factor assembly: $\lambda=\gamma \frac{K_{\mathrm{b}}}{K_{\mathrm{b}}+K_{\mathrm{s}}}$.

Loads become:

$$
F_{\mathrm{b}}=F_{\mathrm{o}}+\gamma\left(\frac{K_{\mathrm{b}}}{K_{\mathrm{b}}+K_{\mathrm{s}}}\right) F_{\mathrm{e}} \text { for bolt }
$$

and:

$$
F_{\mathrm{s}}=F_{\mathrm{o}}-\left[1-\gamma\left(\frac{K_{\mathrm{b}}}{K_{\mathrm{b}}+K_{\mathrm{p}}}\right)\right] F_{\mathrm{e}} \text { for structure. }
$$

If we let $x=l$ in the equations above we come back to the previous ones.

Generally, a high tightening load is good for fatigue life. As we can see, the magnitude of the alternative load on the bolt is reduced (Figure 7 ).

\subsection{Stresses in the bolt (Refs. [1-3])}

The stresses in the screw or stud depend on: the tightening method used, the external loads applied to the assembly and, as seen, the way these external loads are applied.

Generally the traction stress is the most important.

The stress level is also higher right at the thread root diameter.

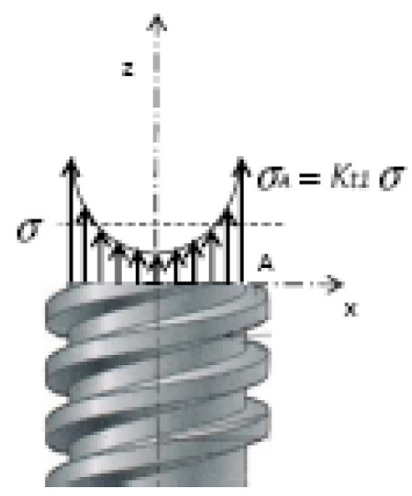

$\mathrm{Kt1}$ is the stress concentration factor for traction

Figure 8. Bolt with only traction stress.
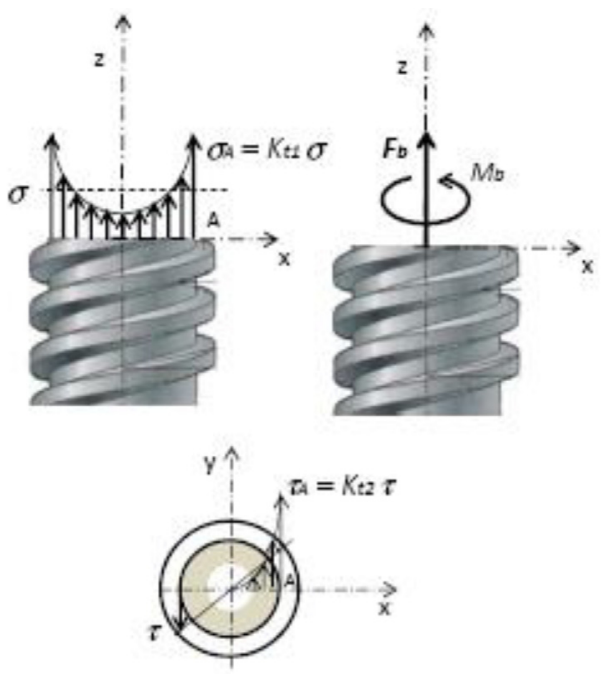

Kt1 is the stress concentration factor for traction $\mathrm{Kt} 2$ is the stress concentration factor for torsion

Figure 9. Bolt with traction stress and torsion stress.

Without torsional stress on the bolt, there is only traction stress (Figures 8 and 9).

On the average it is:

$$
\sigma_{B}=\frac{F_{\mathrm{o}}}{A e} .
$$




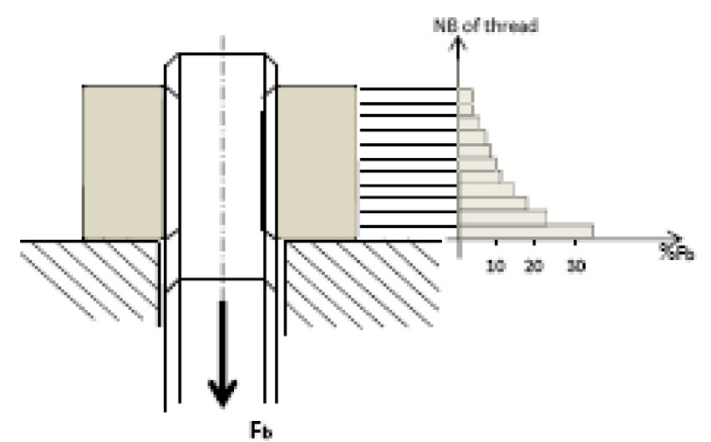

Figure 10. Load distribution in a usual nut.

Ae being the equivalent section of the bolt.

When there is also a torsional stress in the bolt, the equivalent stress is:

$$
\sigma_{e}=\sqrt{\sigma_{B}^{2}+3 \tau_{B}^{2}}
$$

\subsection{In the nut, the distribution of the load among the threads depends on the shape}

Figures 10 and 11 show the load distribution in a usual nut and a suspension nut.

\subsection{Relaxation after tightening}

After tightening there is generally a short term relaxation due to the embedment of the various contact surfaces involved in the joint parts.

Even highly polished surfaces are not perfectly flat; they have some small defects like spots and holes.

When tightened, there will be some local plastic deformations.

This phenomenon lasts even after the tightening operation is completed and all the contact surface areas are large enough to support the bearing contact pressure.

Quite often the operators do not think about this problem.

\subsection{Tightening with a torque wrench (Refs. [1-3])}

This is the most common means to tighten bolts.

Operators do not always know what happens when they carry out the tightening action.

The real preload which is applied to the bolt by torque depends very much on the friction coefficient in threads and the friction coefficient of the contact surfaces between nut and structure.

The relation between the torque and the resulting tightening load is:

$$
T_{\mathrm{T}}=F_{\mathrm{o}}\left(\frac{P}{2 \pi}+f_{1} \cdot r m+f_{2} \cdot \rho m\right) .
$$

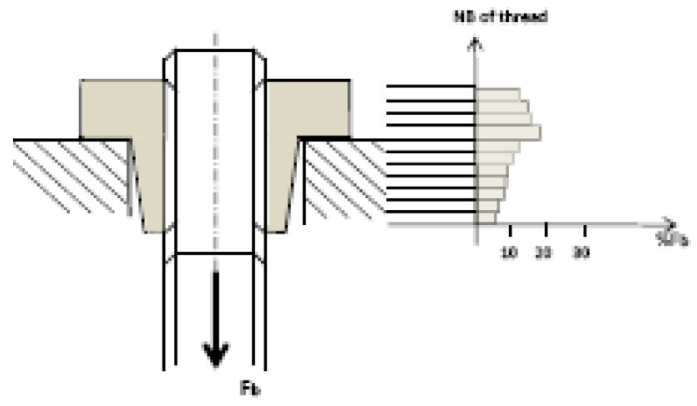

Figure 11. Load distribution in a suspension nut.

To simplify for ISO threads we use the following equation taking into account the standard dimensions of threads:

$$
T_{\mathrm{T}}=F_{\mathrm{o}}\left(0.159 \cdot p+0.583 \cdot d_{2} \cdot f_{1}+\rho m \cdot f_{2}\right),
$$

with:

- $T_{\mathrm{T}}$ applied torque;

- $F_{\mathrm{o}}$ real tightening load;

- $p$ pitch of thread;

- $f_{1}$ and $f_{2}$ friction coefficients respectively for threads and for contact surface between nut and structure;

- $r m$ and $\rho m$ average radius respectively at threads and on contact surface of nut.

The first part of the equation: $T_{\mathrm{th}^{\prime}}=F_{\mathrm{o}}\left(\frac{p}{2 \pi} \cdots\right)$ generates the tightening load in the bolt. for:

It is the "useful" part of the tightening that we should look

The other parts: $T_{\mathrm{th}^{\prime \prime}}+T_{f l}=F_{0}\left(\cdots f_{1} . r m+f_{2} . \rho m\right)$ are only losses due to friction.

It must be pointed out that this "useful" part of the torque is generally only $10-15 \%$ of the total torque.

Even using a sophisticated wrench tool giving a high precision torque $( \pm 5 \%)$ and having low friction coefficients $(0.08 / 0.12$ for threads and $0.10 / 0.15$ for bearing surfaces), the accuracy of the final tightening load on $F_{\mathrm{o}}$ cannot be better than $\pm 20 \%$.

Also, the first two parts of the equation introduces a torsion stress in the bolt which always remains in the bolt, even after the tightening operation is completed.

$$
T_{\mathrm{th}}=T_{\mathrm{th}^{\prime}}+T_{\mathrm{th}^{\prime \prime}}=F_{\mathrm{o}}\left(\frac{p}{2 \pi}+f_{1} \cdot \mathrm{rm} \cdots\right) .
$$

Consequently the stress level in a bolt tightened with a torque wrench can be quite high.

The equations are the following:

Traction stress: $\sigma_{B}=\frac{F_{0}}{A e} ; \quad$ Torsion stress: $\tau_{B}=\frac{16 \cdot T_{\text {th }}}{\pi \cdot d_{e}^{3}}$.

As already mentioned, equivalent stress is shown in equation (6). 


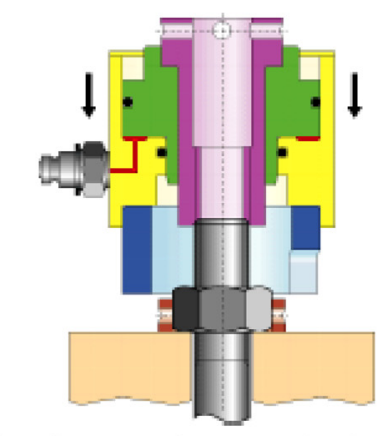

1- The turndown socket is placed over the nut and the hydraulk tensioner graspa the bolt.

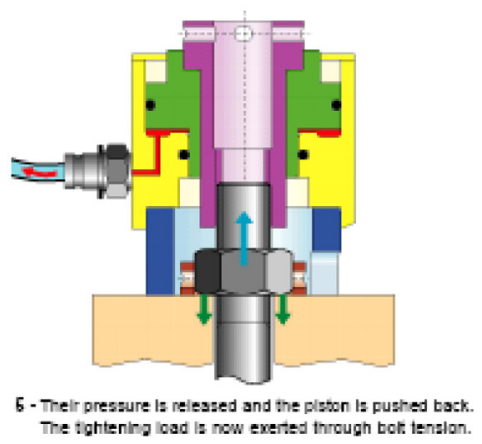

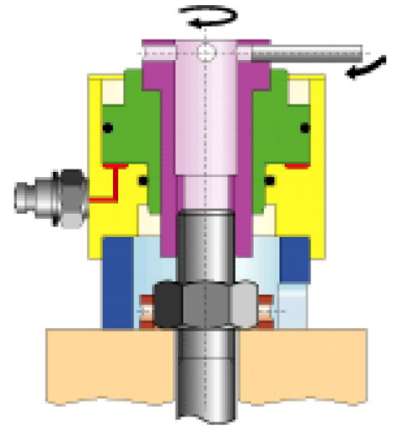

2 - The braceiretract

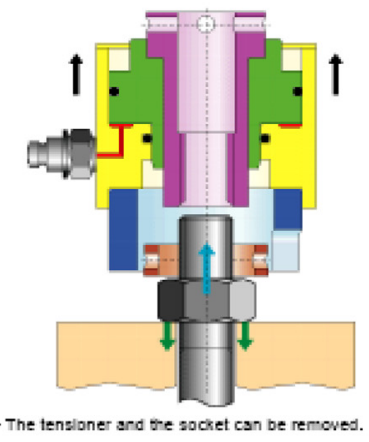

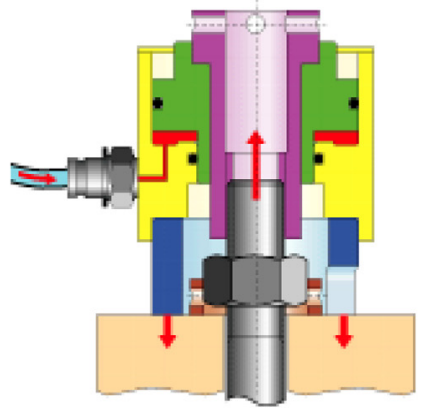

3- After the lydraule consettons, the tens oner is pressrlaed and apples the reculred tractlue force on the bolt.

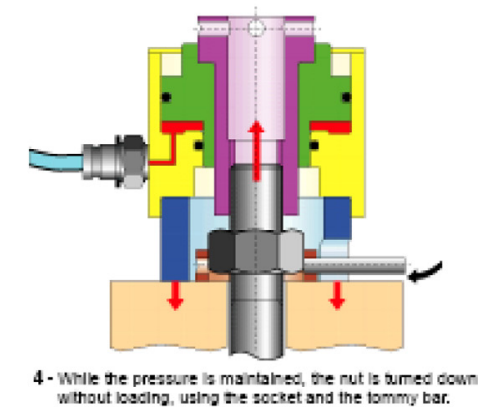

Figure 12. Steps of the tightening operation with a hydraulic bolt tensioner.

Furthermore, the torque wrench can also cause damage on the bearing surfaces due to high friction when the nut is turned under a heavy load.

\subsection{Tightening with hydraulic bolt tensioner (Refs. [1-3])}

Tightening bolt with hydraulic bolt tensioner consists in applying a traction load directly to the bolt to extend it and then to turn down the nut until it is contact with the surface of the structure.

Almost no torque is applied. Process is shown here after on Figure 12 (see Ref. [2]).

Contrary to what many people might think, the hydraulic bolt tensioner can be used to tighten quite small bolts.

Actually, the range of bolt sizes which can be tightened by hydraulic bolt tensioner is very large: from dia. $5 \mathrm{~mm}$ to dia. $500 \mathrm{~mm}$.

With such a tightening mean, there is almost no torsion stress in the bolt after the tightening operation is completed.

The equivalent stress is shown in equation (5).

Bolt tensioner has many advantages such as better accuracy and reliability of tightening load, high tightening load capacity, no torsion stress, no damage on bearing surfaces, easy simultaneous tightening.

But with a hydraulic bolt tensioner, when pressure is released, there is a transfer of the load from the tensioner to the nut. Hence, the remaining tightening load in the bolt $F_{\mathrm{o}}$ is lower than the hydraulic load $F_{\mathrm{h}}$ applied by the tensioner.
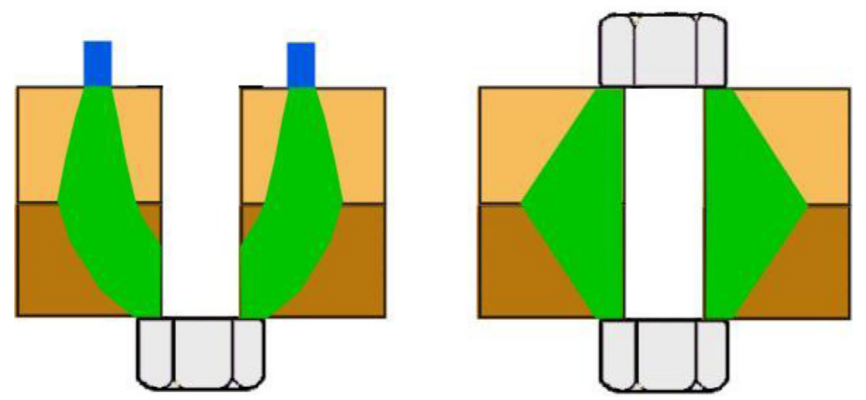

Figure 13. Image of transfer of load from the tensioner to the nut at pressure release.

To know what hydraulic load $F_{\mathrm{h}}$ has to be applied to obtain the required tightening load $F_{\mathrm{o}}$ in the bolt at the end of the operation, we introduce the ratio $F_{\mathrm{h}} / F_{\mathrm{o}}$.

We shall see later that it is possible to overcome this point.

\subsection{Hydraulic bolt tensioner: hydraulic load and tightening load}

Why the final tightening load $F_{\mathrm{o}}$ is lower than the hydraulic load $F_{\mathrm{h}}$ applied by the bolt tensioner?

The reason for the difference between $F_{\mathrm{h}}$ and $F_{\mathrm{o}}$ is that there is a transfer of load applied by the tensioner to the nut when the pressure is released (Figures 13 and 14).

This leads to a deformation of the threads of bolt and nut in connection, a deformation of the nut body and a compression of the structure right under the nut. 

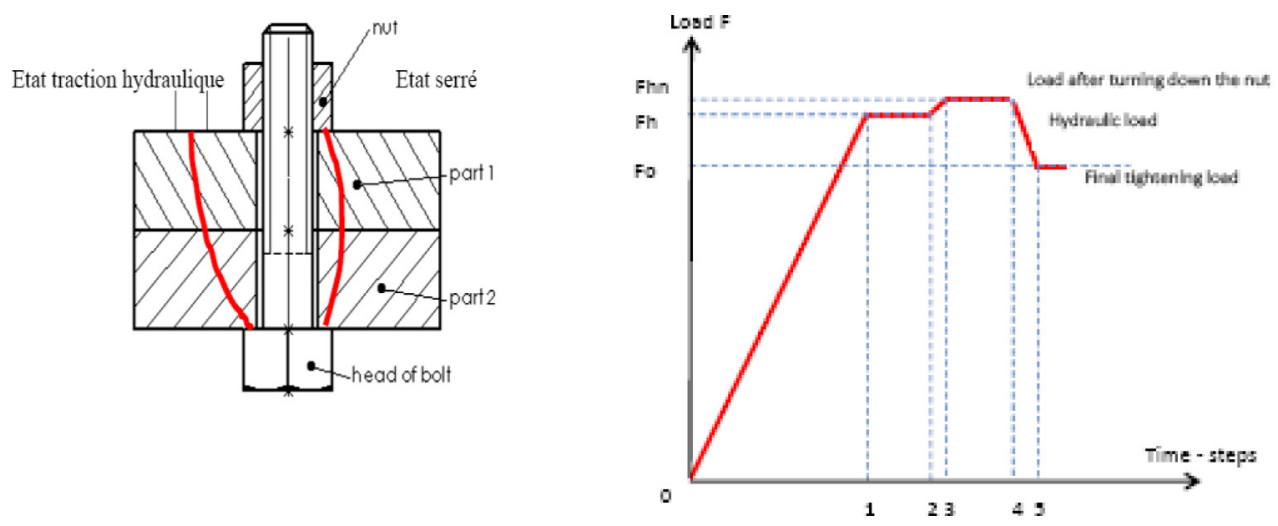

Figure 14. Graph showing the evolution of the load on bolt when tightening with a tensioner.

Three possibilities to obtain the tightening load :

1. Use the simplest but less accurate $F_{\mathrm{h}} / F_{\mathrm{o}}$ graph.

2. Make real tests on the application or on mock up with sensors.

3. Make cumbersome calculations.

\subsection{1 $F_{h} / F_{o}$ graph}

A graph based on long experience on several thousand cases and commonly used in the profession (Figure 15).

It gives the ration $F_{\mathrm{h}} / F_{\mathrm{o}}$ depending on the ratio $L / d$ (tightened length/diameter).

It is simple but not very accurate.

For example for a $\mathrm{M} 20 \times 2.5$ bolt $200 \mathrm{~mm}$ long, the ratio $F_{\mathrm{h}} / F_{\mathrm{o}}$ is given with a large tolerance: $1.12-1.22$, or: $1.17 \pm 0.5$ $( \pm 4.5 \%)$.

This general graph takes into account the various types of assemblies, shapes and properties of parts, in particular the threads. However, the upper limit of the graph (orange) applies mainly to large pitches while the bottom limit (green) is mainly for small pitches.

Standard pitches lie in the bottom third of the graph.

\subsubsection{Real tests on application or on mock up with sensors}

This is the most accurate way to obtain the real final tightening load $F_{\mathrm{o}}$ (Figure 16).

However, it is also the most expensive.

It requires time, additional equipment, calibration tests, further machining and must be carried out by specialists.

Possibly, not all the requirements could be implemented.

\subsubsection{Cumbersome calculations}

This can provide good accuracy and does not require additional time and money after the development has been made.

The development, which has been carried out by Dr. Habib Karaouni, has resulted in what we call "Optimum Tightening Process (OTP)" for bolt tensioner.
It is based on the Zarka's "Intelligent Optimal Design (IOD)" (Ref. [4]).

A tool has been created which allows calculating the two cases: tightening of a single bolt assembly and tightening of a multiple bolt assembly.

The following part of the presentation gives some details on the development.

\section{Part II: intelligent optimum design - application to bolt tightening}

The application of IOD is made preferably to tightening with bolt tensioner because, as mentioned above, this mean has numerous advantages and it is now more and more used, in particular when tightening requirements are quite stringent.

\subsection{Intelligent optimum design - general methodology (Ref. [4])}

As already indicated, this method has been developed by J. Zarka and his team in their book (see Ref. [4]).

“We don't know everything!

Nevertheless, it is necessary to provide answers to our problems in a practical and optimal manner.

Engineers face important problems during the design phase, tests, monitoring and maintenance of structures. Not even experts in the field have all the answers to these problems (such as no satisfactory constitutive modelling of materials, no real control of the accuracy of the numerical simulations, no real definition of the initial state and/or the effective loading of the structure, etc.), There is no perfect solution as the experts do not understand the problem in its entirety. Moreover, the available data may not be statistically representative (i.e. limited in number), and may even be fuzzy, qualitative and/or partly missing. This implies that other methods need to be used. 


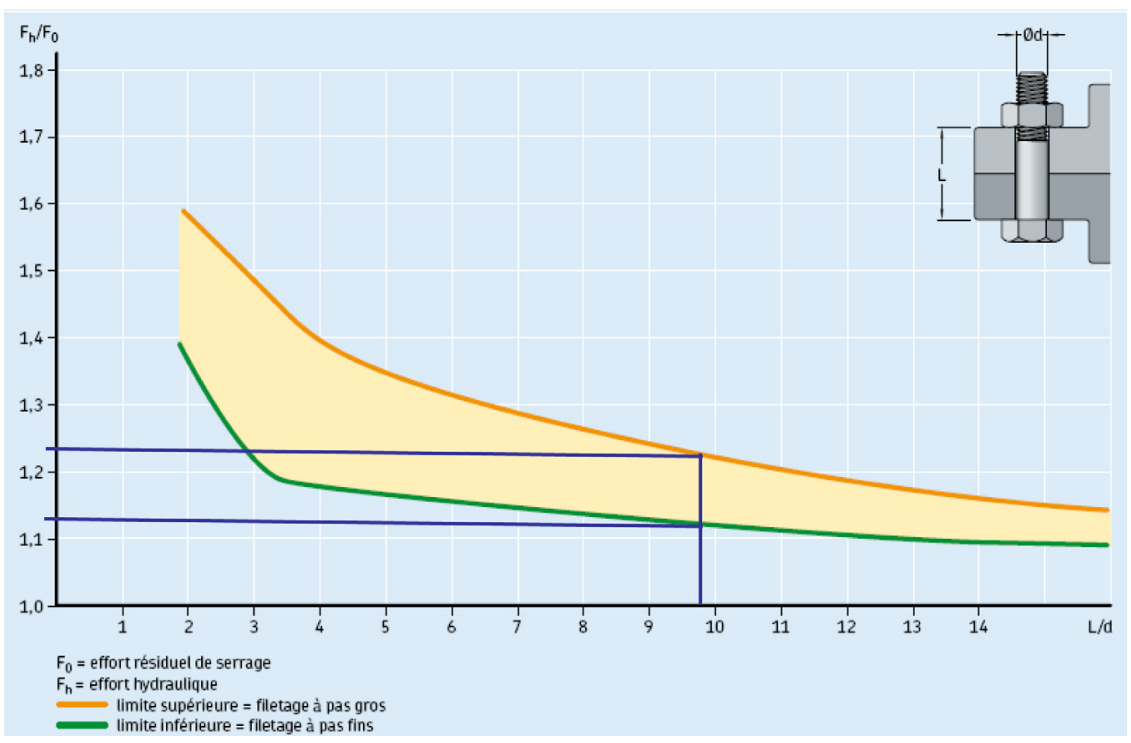

Figure 15. Graph $F_{\mathrm{h}} / F_{\mathrm{o}}$ based on long experience.

Hence the use of a combination of new Artificial Intelligence (AI) and Multidisciplinary Optimization.

All the information available in the company concerning the problem can be collected and used.

Any form of information is useful: material (results of experiments, experience feedback, results of numerical simulations, etc.) or immaterial (general knowhow, state-of-the-art, knowledge of the experts, etc.).

Information is in fact rather haphazard.

Even if this Information is in fact rather haphazard, it is the raw material of the IOD."

\subsubsection{Intelligent optimum design method based on automatic learning expert systems}

Nowadays, automatic learning generators are able to automatically extract the rules from the raw examples base defined by the expert. The experts are aware that they do not know the full solution, but are able to build an examples base, for which the solution is known experimentally or numerically. Their aim is to provide a good description of such an examples base.

The automatic learning system involves five main functions:

Prepare: To transform the example files from user format (ASCII, excel, etc.) into the specific format of the system and to handle the discretization of the descriptors and the splitting of the initial data base into a training set and a test set.

Learn: To automatically extract a rules base from the training set according to the quality of available information (noise, sparseness of the training set, etc.) using Statistical, Symbolic, Numerical, Fuzzy logic, Neural Network or Genetic Algorithm learning.

Test: To experimentally evaluate the quality of the extracted rule on the test set.

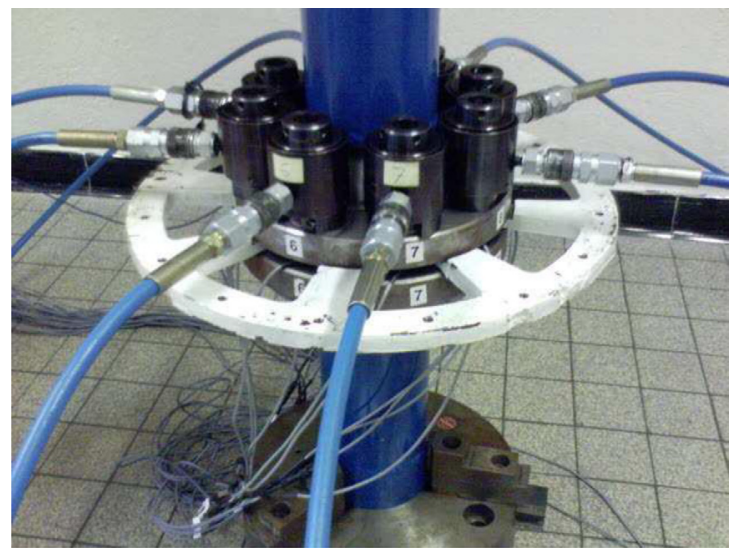

Figure 16. Example of measurement of the final tightening load $F_{\mathrm{o}}$ on a bolted assembly.

Inclear: To allow the expert to visualize IN CLEAR the extracted rules with the user format and to state what descriptors are the relevant pertinent descriptors to be kept.

Conclude: From the description of a new case, to deliver a conclusion based on the extracted rules.

\subsubsection{The basic stages of the method are the following}

\section{BUILDING THE DATA BASE}

EXPERTS $=>$ all variables or descriptors which may take a part

PRIMITIVE descriptors XX (large number) with the actual whole knowledge, simplified analytical models, simplified analysis, complex (but insufficient) beautiful theories!!

INTELLIGENT descriptors X (limited number) 

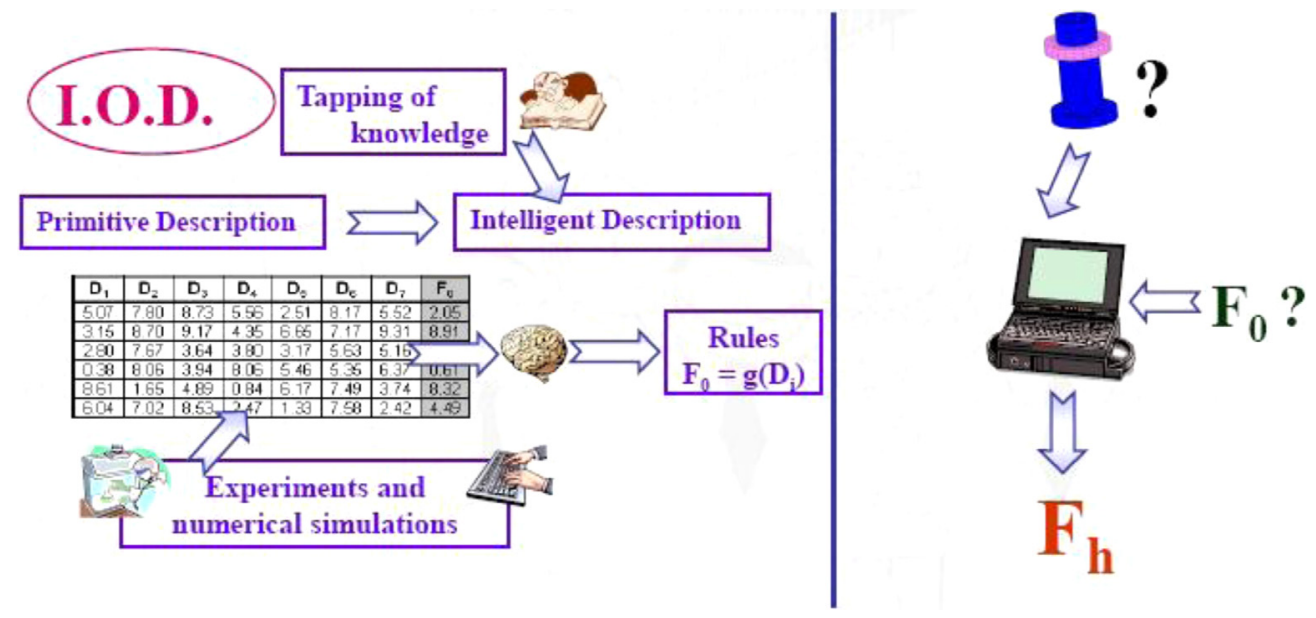

Figure 17. Figurative presentation of IOD applied to bolt tensioner.

\begin{tabular}{ll}
\hline Input descriptors & Output descriptors or conclusions \\
\hline Number & Classes (good, not good, leak, break, etc.) \\
Boolean & Numbers (cost, weight, etc.) \\
Alphanumeric & $\rightarrow 50$ examples in the data base \\
Name of files & $\rightarrow 10-1000$ descriptors \\
Data base access & $\rightarrow 1-20$ conclusions \\
Curves, signals, & MOST IMPORTANT (DIFFICULT) TASK \\
\multicolumn{1}{c}{ pictures, etc. } & $(99.99$ of the work !!!!!!) \\
\hline
\end{tabular}

2. GENERATING THE RULES with any Machine Learning tool

Intelligent descriptors help the algorithms.

Each conclusion explained as a function or rules of some intelligent descriptors with known reliability.

If too low: this means not enough data, bad or missing intelligent descriptors.

\section{APPLICATION TO NEW EXAMPLES}

4. OPTIMIZATION at two levels (Inverse Problem)

(i) Independent intelligent descriptors

$$
\begin{aligned}
& =>\text { may be impossible OPTIMAL SOLUTION } \\
& =>\text { but DISCOVERY OF NEW MECHANISMS }
\end{aligned}
$$

(ii) Intelligent descriptors linked to primitive descriptors

$$
\begin{aligned}
& =>\text { OPTIMAL SOLUTION } \\
& =>\text { technologically possible! }
\end{aligned}
$$

\section{2 "Optimization of tightening process" to tightening operation of a single bolt with bolt tensioner}

The problem can be shown in Figure 17.

As already mentioned, we want to know the ratio $F_{\mathrm{h}} / F_{\mathrm{o}}$ simply by giving some input to the system.
The selected tightening means is the hydraulic bolt tensioner.

The first analysis is made for the case of a single bolt tightening.

Depending on the situation the problem can have two different aspects:

- either to find out which will be the final tightening load $F_{\mathrm{o}}$ after applying the hydraulic load $F_{\mathrm{h}}$, this is what we call the "Direct problem";

- or to define which hydraulic load $F_{\mathrm{h}}$ needs to be applied to get the required tightening load $F_{\mathrm{o}}$, this is what we call the "inverse problem".

\subsubsection{Direct problem}

Getting $F_{\mathrm{o}}$ when known $F_{\mathrm{h}}$ is applied by the bolt tensioner (Figure 18).

\subsubsection{Inverse problem}

Getting necessary $F_{\mathrm{h}}$ by the bolt tensioner for correct $F_{\mathrm{o}}$ at end of operation (Figure 19).

As indicated above the method implies to define "Primitive Descriptors" and then a limited number of "Intelligent Descriptors".

\section{- Primitive descriptors}

There are many primitive descriptors because many parameters must be considered.

Geometrical data of the structure and the bolt (all dimensions and gaps), mechanical properties of the various parts of the structure, mechanical properties of the bolt and nut, friction coefficients of contact surfaces and dimensions and properties of the bolt tensioner (Figure 20).

\section{- Input descriptors}

Geometric descriptors of the bolt (screw \pm nut) Geometric descriptors of fastened structure 


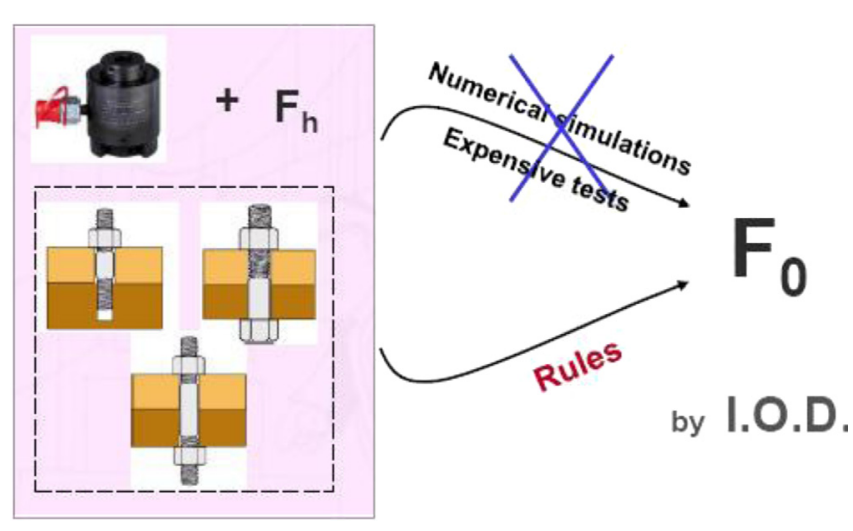

Figure 18. Figurative presentations of direct problem.

Geometric descriptors of the tensioner

Material descriptors of the bolt (screw \pm nut)

Material descriptors of fastened structure

Tribology descriptors of assembly

Loading descriptor of assembly

\section{- Output descriptors}

Four conclusions descriptors have been taken.

\section{- Intelligent descriptors}

We have selected three categories of intelligent descriptors

Intelligent descriptors 1st category (structural engineering)

Any value which has some meaning in terms of physics, comes directly from knowledge of experts and does not represent a direct estimation of the response to the problem.in

Intelligent descriptors 2nd category (structural engineering)

Giving a direct estimation of the response to the problem. This can be obtained either from analytical solutions or from automatic learning of the response of a sub-problem.

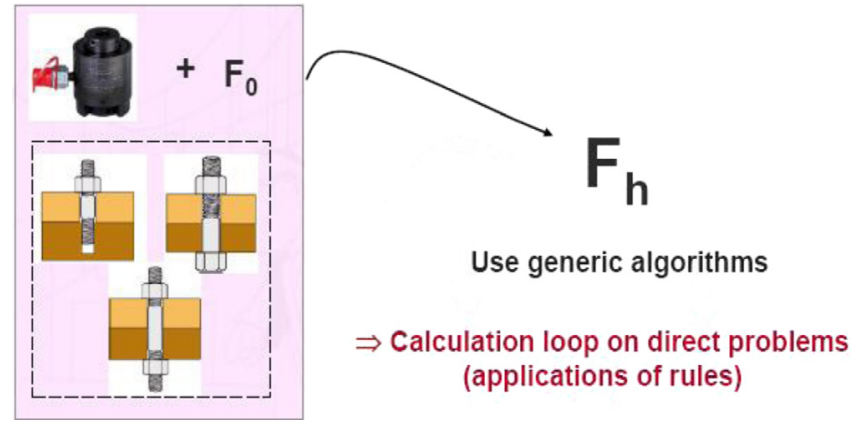

Figure 19. Figurative presentations of inverse problem.

Intelligent descriptors 3rd category (structural engineering) Intermediate values which may not have a meaning in terms of physics but which can help expert learning systems to define rules.

\section{- Building the data base}

To generate the data base we use experimental and on-site results as well as cases generated by numerical simulation. It represents the longest and most difficult part of the method.

Several cases have been created to have a significant statistical representation of the input parameters to see the impact of all parameters as well as the interactions between them. To carry out this task the following calculation loop is followed (Figure 21).

At this step we need to remember point 7 here before "precautions when tightening" where we mentioned possible tight fit in the holes and short term relaxation which lasts even after the tightening operation is over.

To overcome these problems when using a hydraulic bolt tensioner, a double turning down of the nut is carried out.

The process is as follows (see Figure 22):

- installation of the tensioner and screwing of the brace on protruding part of the bolt;

- first pressurization is to apply $F_{\mathrm{h}}$ load for extension of bolt;

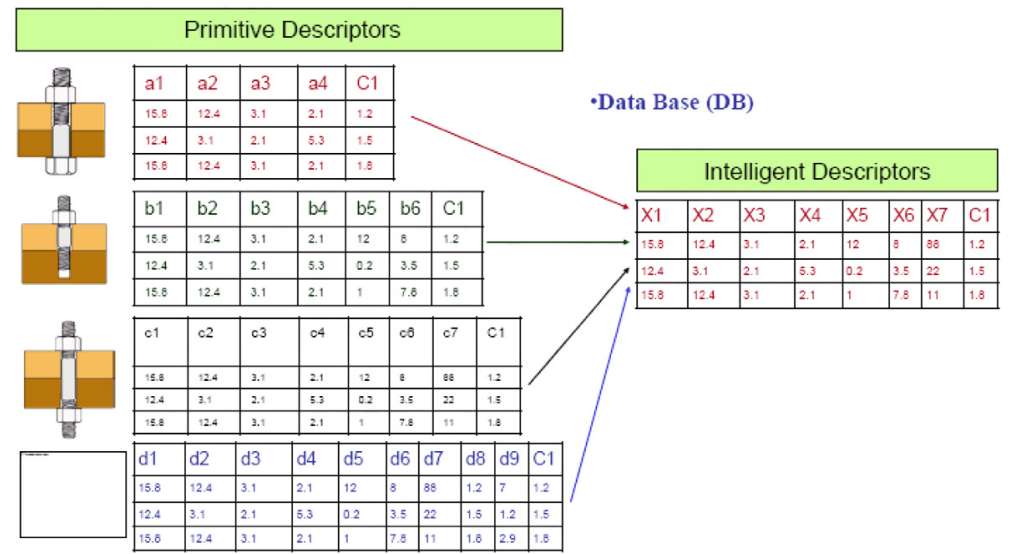

Figure 20. Example of a table of primitive descriptors. 


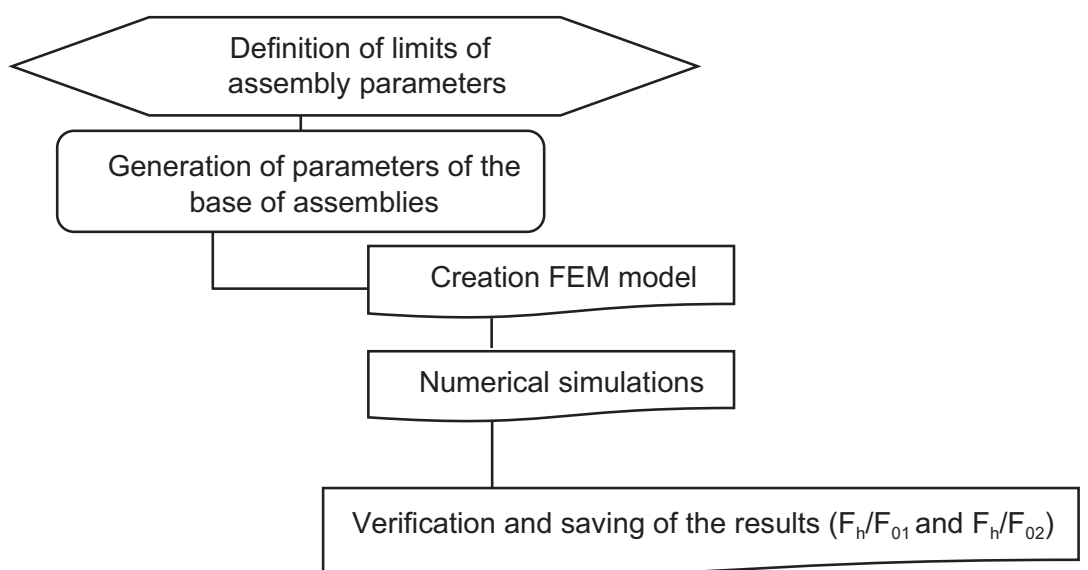

Figure 21. Flow chart of creation of the data base.

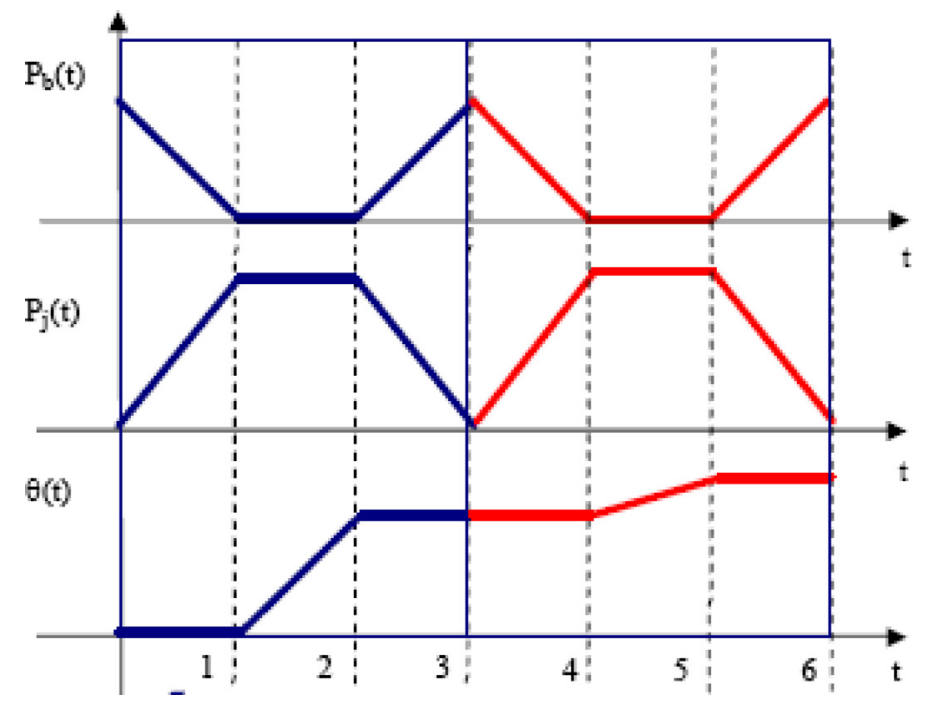

$0-1$ : first pressurization

1-2: first turning down of the nut

2-3: first pressure release

3-4: second pressurization

4-5: second turning down of the nut

5-6: final pressure release

Figure 22. Illustration of the loading sequence on bolt.

- first turning down of the nut;

- pressure release;

- second pressurization is to apply again $F_{\mathrm{h}}$ load for extension of bolt;

- second turning down of the nut;

- final pressure release;

- removal of the tensioner.

This reduces the incidence of tight fit in the hole and almost eliminates the short term relaxation by making an early embedment of contact surfaces.

This nut double turning down process has been taken into account in the OTP.

\section{- Generation of the base of assemblies}

More than 700 bolted assemblies have been generated in addition to the real cases.

Among them are:

- $15 \%$ technology cases;
- $1 \%$ "extreme" cases;

- $84 \%$ random controlled cases.

\section{- Automatic creation of FEM model}

A software tool has been created by $M Z$ to generate, automatically, the driving files of the FEM models of the base of assemblies. All the following parameters are taken into account: the geometry of the assembly and the tensioner, the materials, the definition of the meshing, the internal contact surfaces and the selected loading.

\section{- Loading process on the assembly}

To simulate the loading on the bolt a field of uniform temperature is applied. The magnitude is such that the final elongation of the bolt is the same as when exerting the traction load and the sign shows whether it is traction or release (Figure 23). 


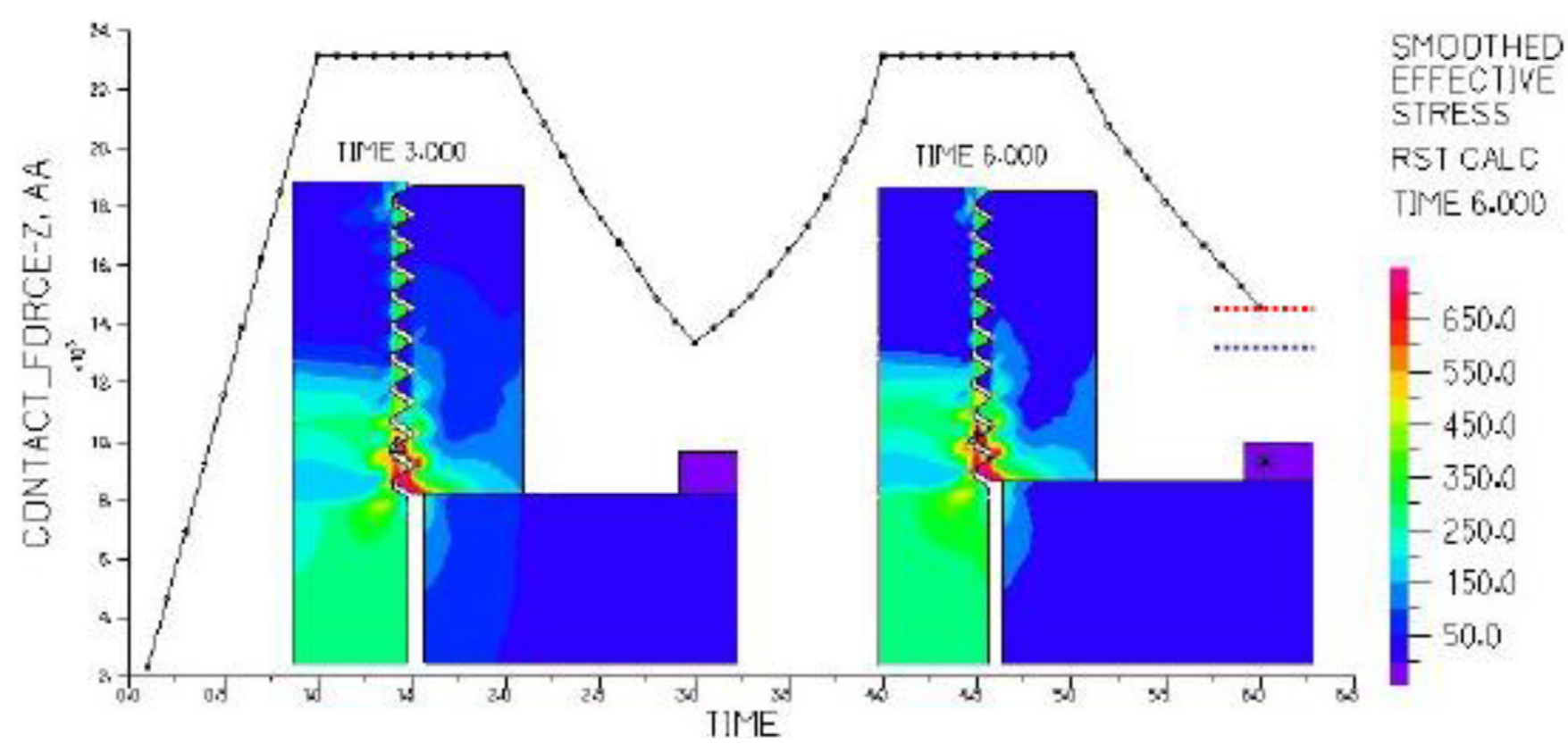

Figure 23. Display of load on assembly and Von Mises stress in the thread during tightening sequence.

The turning down of the nut is simulated by applying different temperatures to the bolt.

\section{- Generation of the rules}

The data base has more than 700 cases. Each is described by 33 input descriptors and four output descriptors.

The software used for the learning are SEA of LMS-X and SeaTools from MZ.

The learning process involves the following:

- Learning the 1 st turning down of the nut $\left(F_{\mathrm{h}} / F_{01}\right)$

- First, make the learning of a sub-problem on a sub-base. This sub-base contains about 200 carefully selected samples to represent the boarder of the domain of definition. This first learning phase shows the good impact of the parameters.

- Next, make the learning of the primary problem using the help of the prediction of the previous subproblem.

- Learning the 2 nd turning down of the nut $\left(F_{\mathrm{h}} / F_{02}\right)$

- Use the results given by the prediction of the first turning down since the prediction has a good reliability and good robustness.

It is only when there is reliability, efficiency and robustness of the solution for all these cases that the validity of the rules is confirmed.

Generally several iterations and sometimes additional intelligent descriptors are necessary to reach a valid and reliable solution.

Figures 24a and 24b show optimal results obtained after running the learning process several times.

\section{- Building the autonomous tool}

This process allows the building of a system that is able to predict the ratio $F_{\mathrm{h}} / F_{0}$ for any single bolted joint with a large variety of bolt geometries, structures and tensioners and various mechanical properties of concerned materials.

Figure 25 shows the window screen to input data.

Figures 26a and 26b show the window screen of the results. On first picture we see where ratio $F_{\mathrm{h}} / F_{\mathrm{o}}$ is located on the graph after first turning down of the nut (blue dot) and after second turning down of the nut (red dot).

\subsection{Multiple tightening - multi bolt joint (see Ref. [2])}

After a tool for a single bolt joint has been created, it was necessary to deal with joints with multiple bolts.

The aim is to have an additional tool which defines the better process to guarantee an even distribution of the tightening load on the bolts and to minimize the operating time taking into account the number of tensioners which can be used for the operation and the requirements in terms of tolerance.

This second step of development concerns assemblies with circular flanges joint together by several bolts equally located on a pitch circle diameter.

Several hydraulic bolt tensioners are required for the tightening process.

\subsubsection{Multiple tightening - multi bolt joint - full simultaneous tightening (see Ref. [2])}

Full simultaneous tightening is made when there are as many tensioners as bolts to tighten (Figure 27). 
(a)

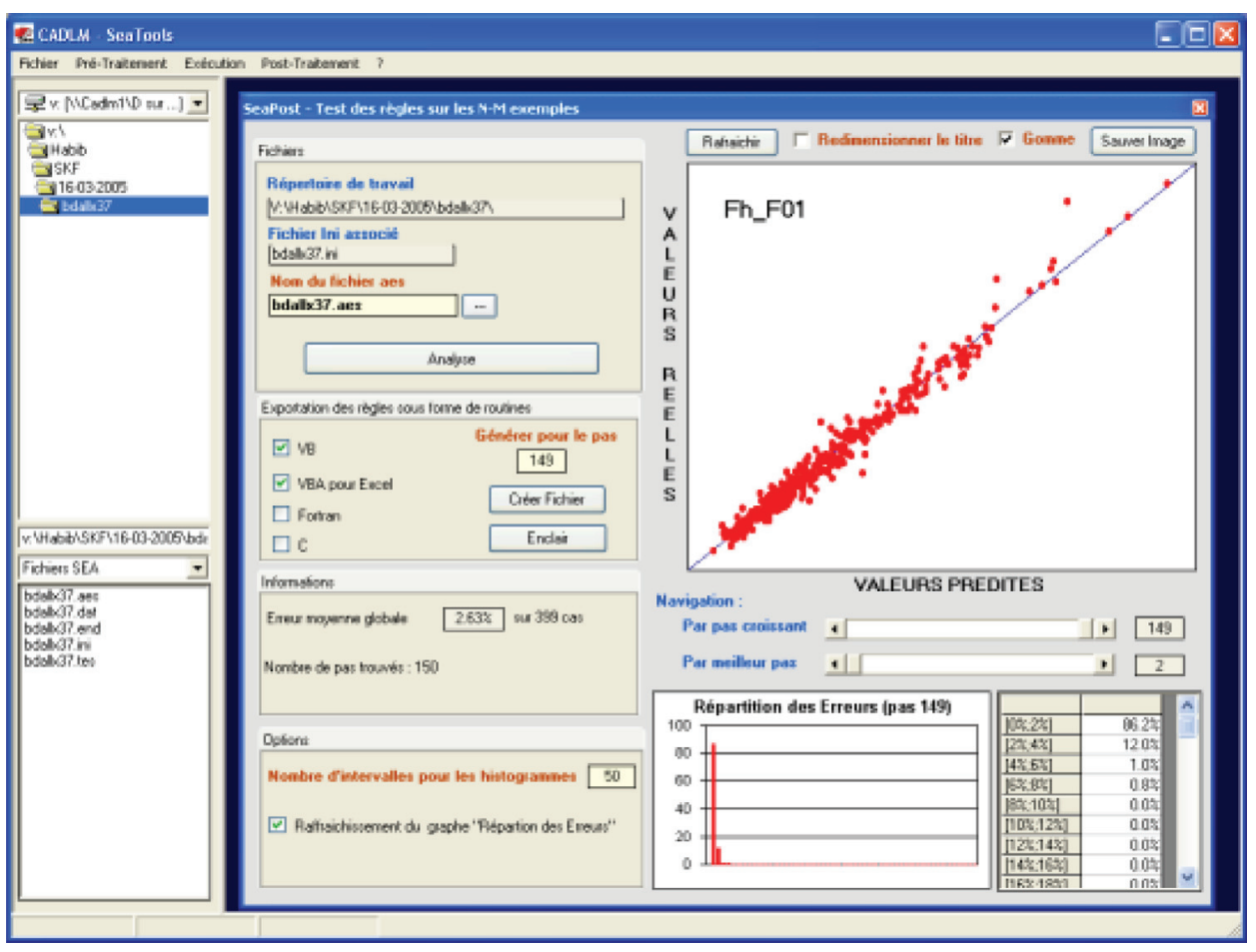

(b)

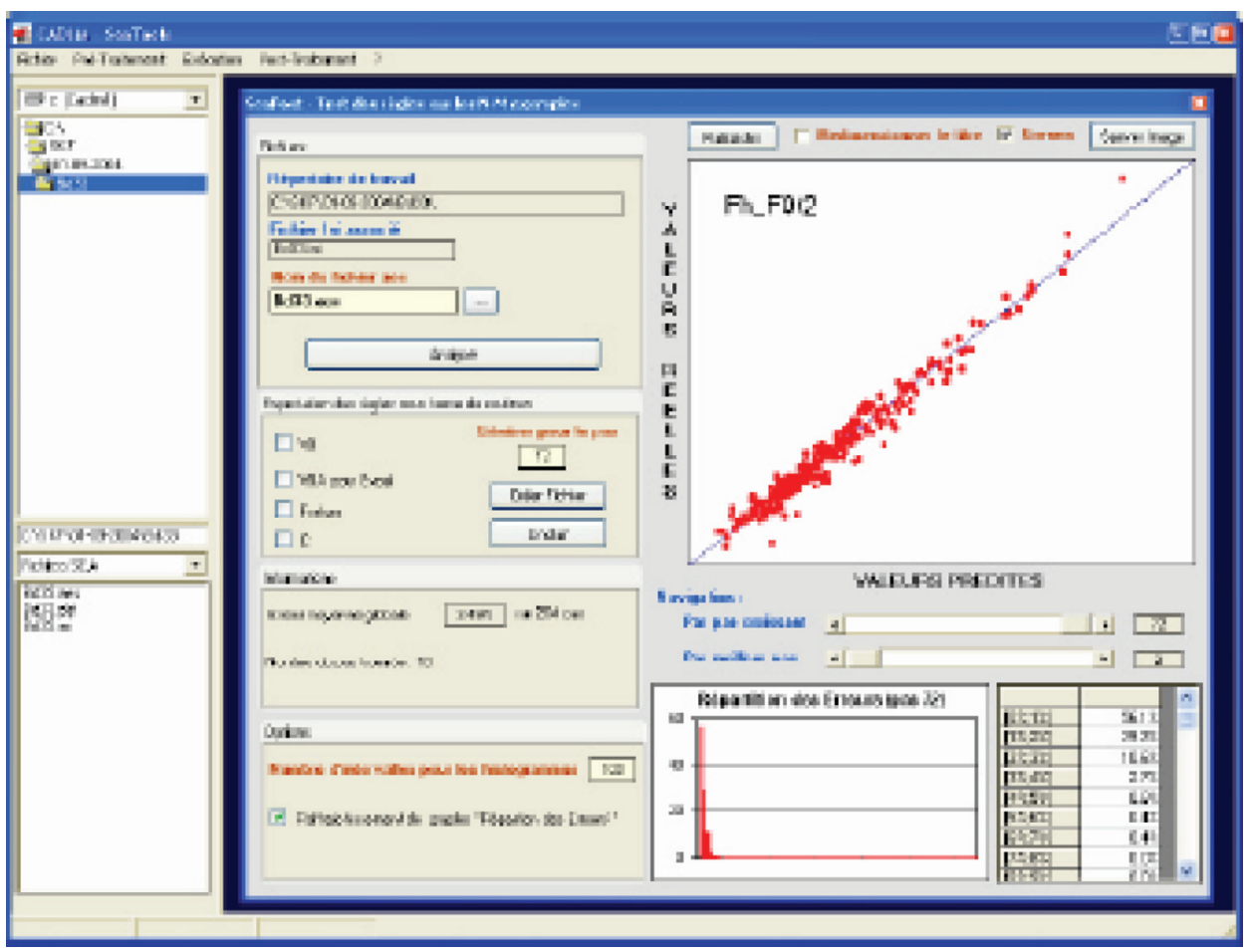

Figure 24. Pictures showing the results of the learning process after first (a) and after second (b) turning down of the nut Von Mises stress in the thread during tightening sequence. 


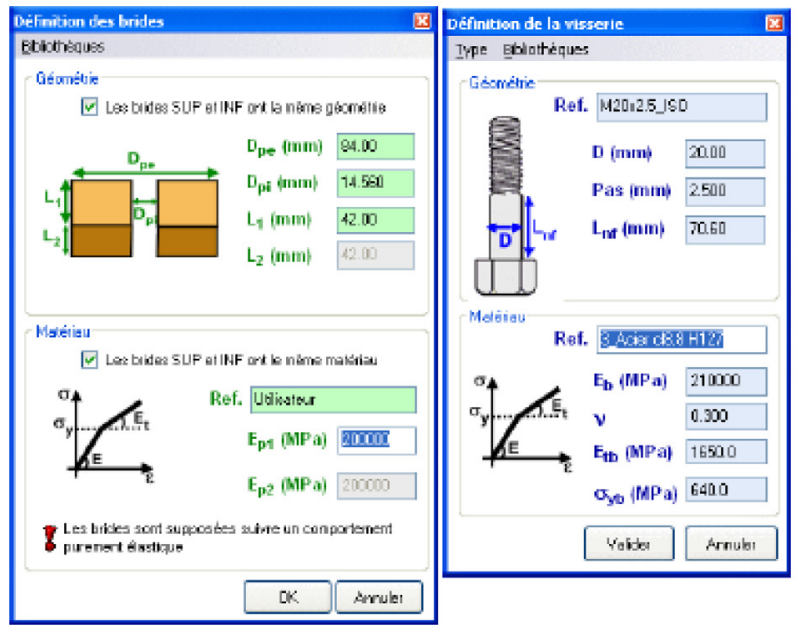

he same time.

It is the most accurate and quickest method but appears expensive at first glance.

However, for many cases, this offers the best solution, after making detailed and complete analysis on determinant factors such as requirements in accuracy, operating time, number of operators involved, handling operations, control operations, cost to follow up parts and components and various kinds of other risks (Figures 28 and 29).

\subsubsection{Multiple tightening - multi bolt joint - non full simultaneous tightening (see Ref. [2])}

When there are fewer tensioners than bolts in the assembly several steps and passes are necessary.

Only a defined number of bolts can be tightened at the same time depending on the number of tensioners.

This is a rather current situation since full simultaneous tightening is quite expensive.

The condition is however to have enough tensioners to make axisymmetric tightening at each pass.

For example 2 tensioners for 4 bolts, 2 or 4 tensioners for 8 bolts, 3 or 4 or 6 tensioners for 12 bolts, etc.

When a set of bolts is tightened, all the other bolts which have been already tightened are impacted.

The closer they are to the bolts in tighten, the more impacted they are as there are interactions between the bolts (Figure 30).

\subsection{Application of the "optimization of tightening process" to multi bolt tightening with bolt tensioners}

Several remarks can be made here.

- First the same types of descriptors as for single bolts are used.

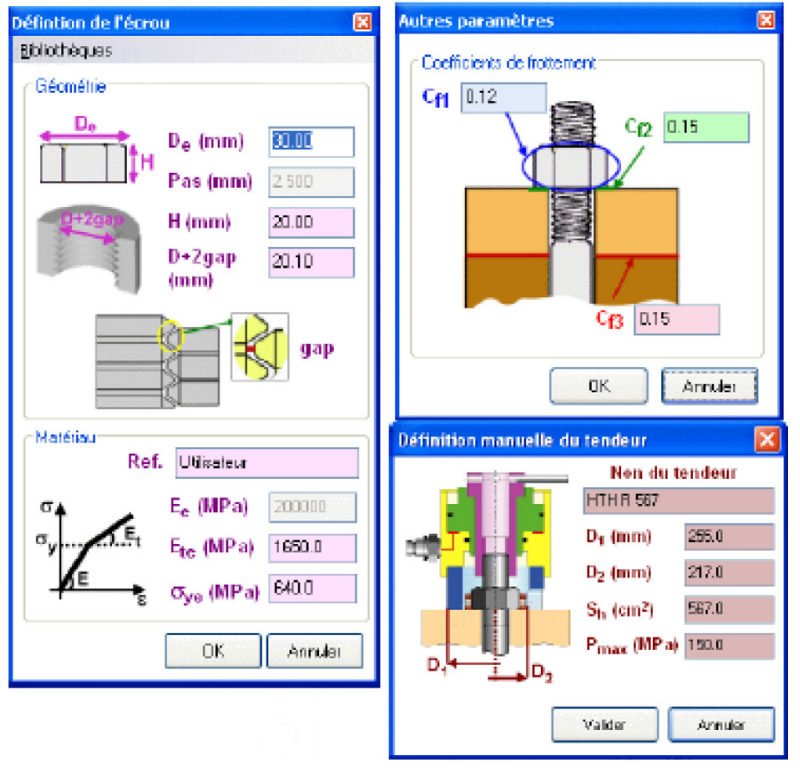

- Only the inverse problem is really relevant in that case.

- There is no difference in nature between full simultaneous tightening and non-full simultaneous tightening, the first one is simply quicker, easier and more accurate than the second one, but the system can deal with both of them.

For non-full simultaneous tightening the system will proposes an optimal process which allows a minimum number of passes should be used.

The system should give the tightening parameters to be applied to each set of bolts and each pass, taking into account additional practical requirements such as the maximum admissible load on bolts, the load capacity of the tensioners and various scenarios which can be followed.

The system works also for full simultaneous tightening.

\subsubsection{The methodology}

When tightening a bolt, its tightened length is reduced. The tightening load depends on the interaction with the joint members (flanges). If the length of the bolt is the same as the thickness of compressed flanges, the resulting force between action load and reaction load is nil.

This is also true for multiple tightening. At each stage of the tightening process the length of each bolt is the same as the corresponding compressed part of flanges. The load in each bolt depends on the initial length.

Knowing this, the load $F_{\mathrm{o}}$ can be calculated at each step and $F_{\mathrm{h}}$ is obtained by means of the ration $F_{\mathrm{h}} / F_{\mathrm{o}}$ which is given by the tool already presented here above.

Two approaches can be made.

The first one consists in representing both cases, single bolt and multiple bolts, by parallel bars between thick plates. The big bar represents the flanges and the small bars represent the bolts (Figure 31). 
(a)

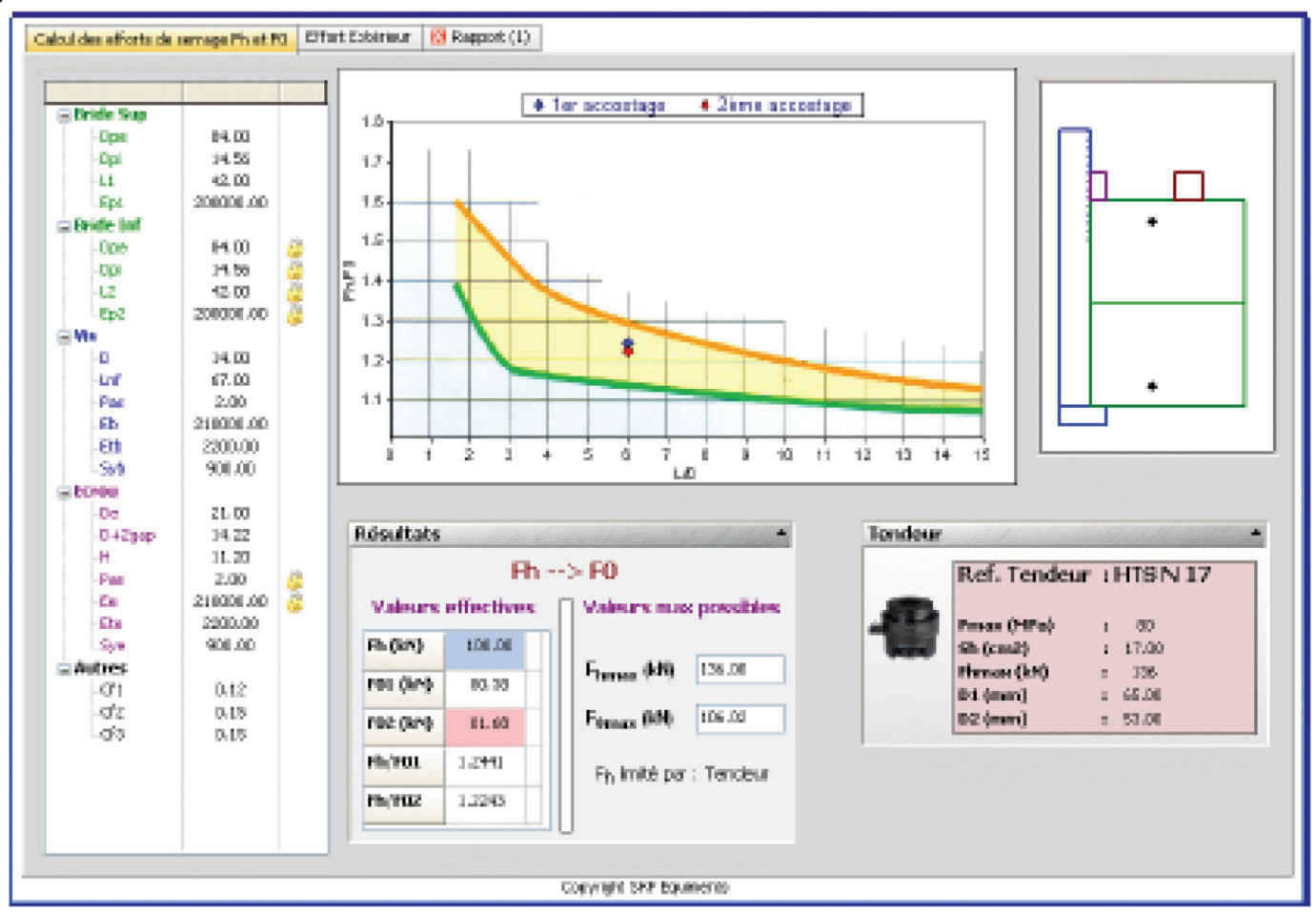

(b)

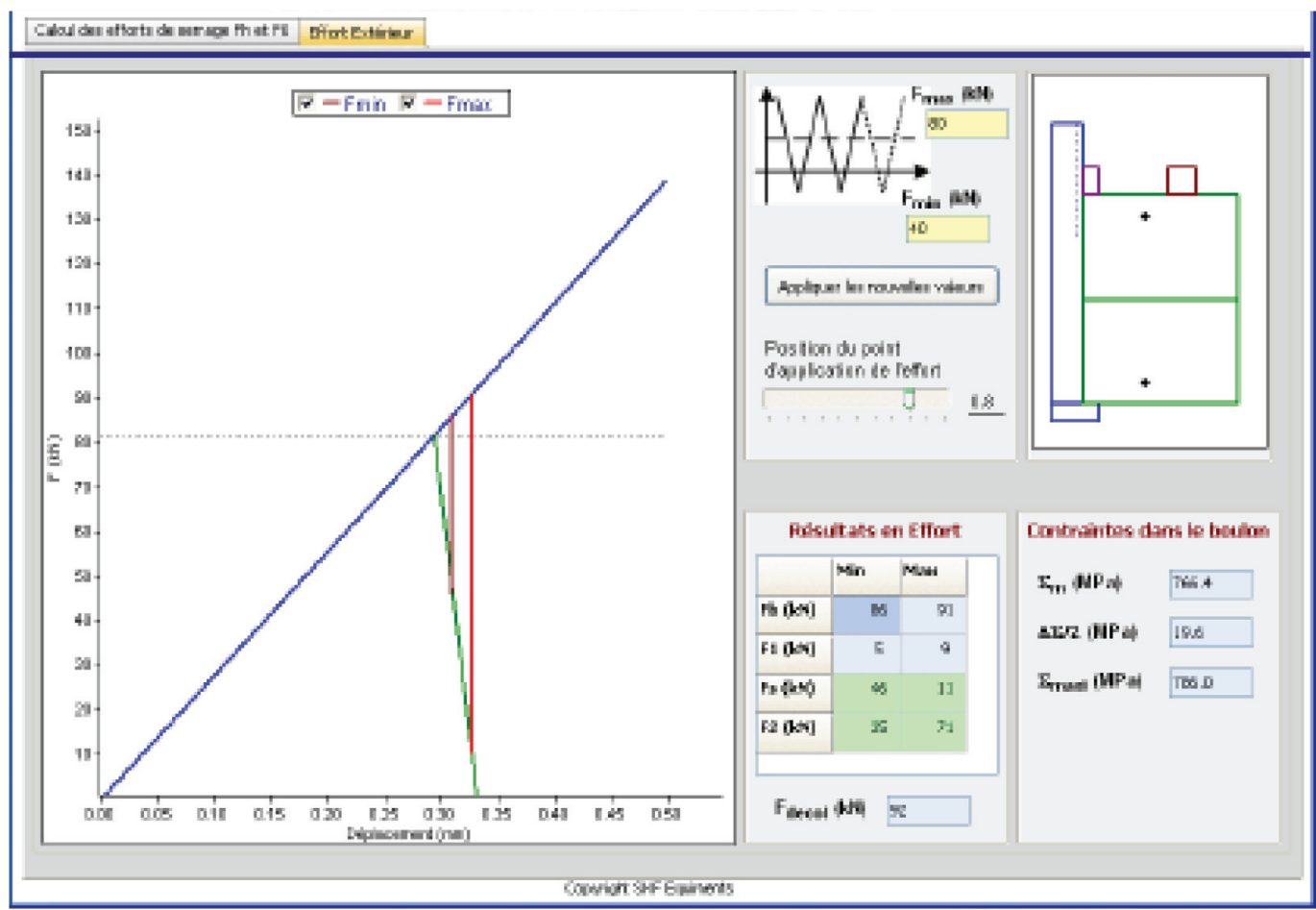

Figure 26. (a) Window screen of results giving the ratio $F_{\mathrm{h}} / F_{\mathrm{o}}$, values and location on the graph. (b) Windows showing the way the results are displayed: application of an external load. 

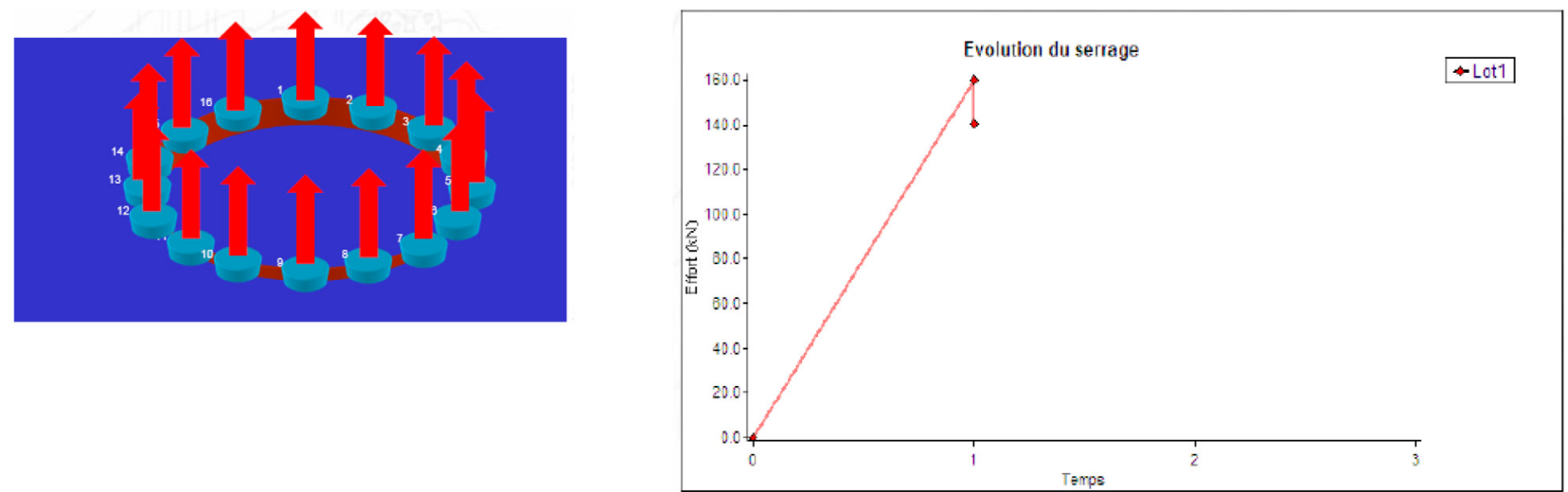

Figure 27. Picture and graph showing full simultaneous tightening.
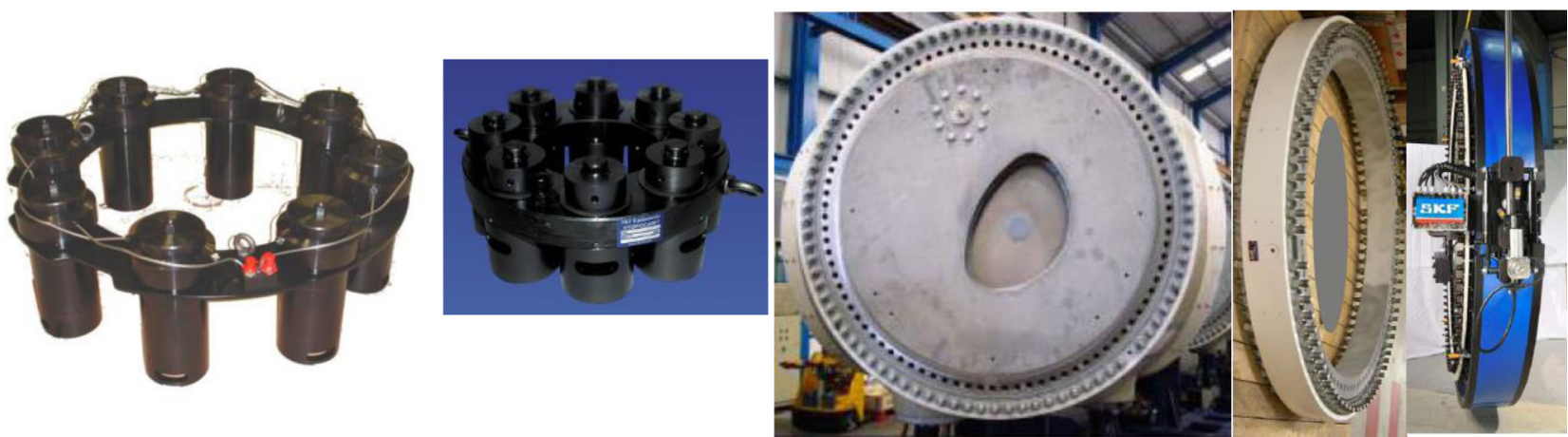

Figure 28. Examples of full simultaneous tightening: diesel engine top head, petro-chemical pipe junction, and slewing bearing of wind turbine blade.

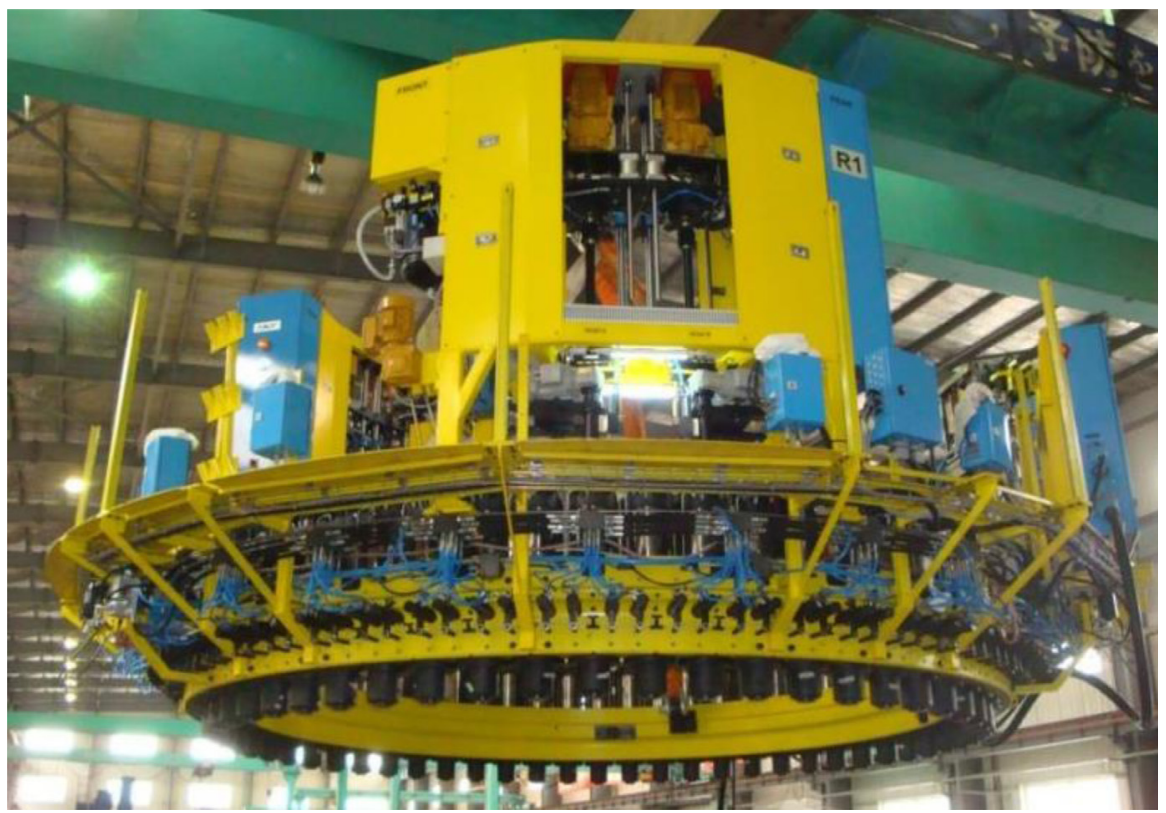

Figure 29. Other example of full simultaneous tightening: Multiple Stud Tensioning Machine (MSTM) for PWR nuclear reactor. 


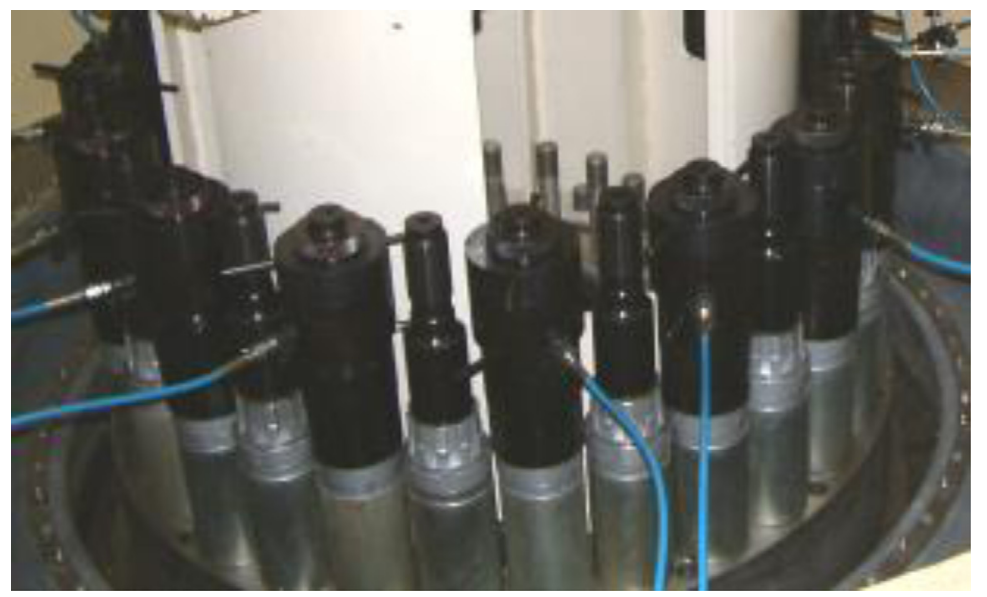

Figure 30. Example non-full simultaneous tightening (here one-bolt-in-two).

(a)

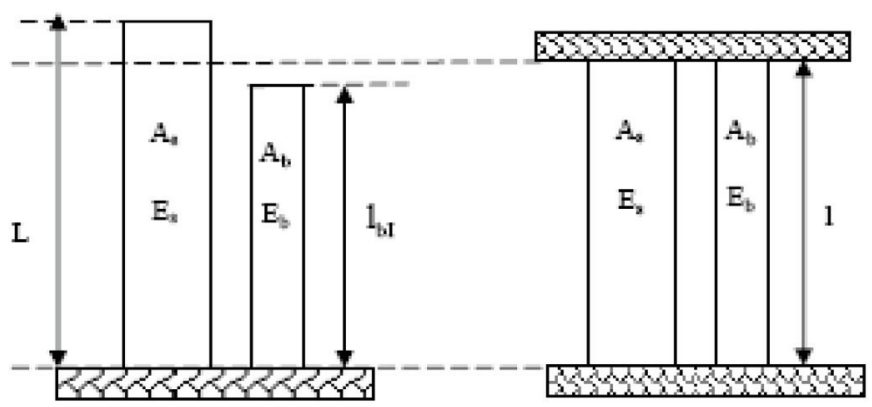

Before tightening

After tightening

(b)

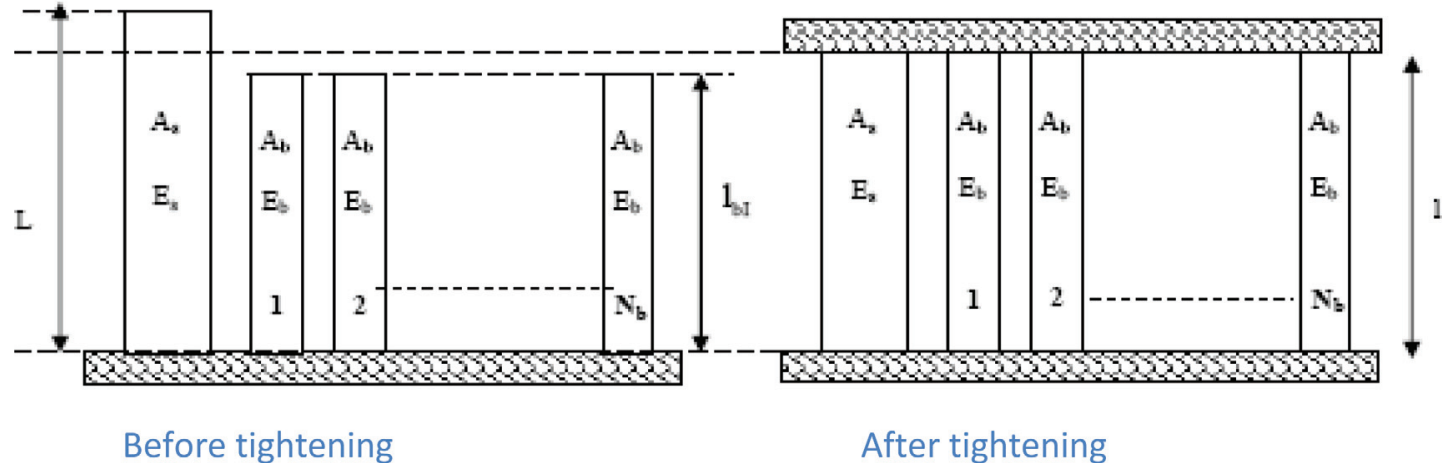

Figure 31. Illustration of the bar system to represent the flanges and the bolts. (a) Single bolt, (b) multi bolt.

For the case of multi bolts, the bar 1 represents the first set of bolts tightened together, the bar 2 represents the second set of bolts tightened together and so...

The second approach consists in the calculation of a coupling matrix which represents the impact of the tightening of each set of bolts on the sets.

Both approaches have been analysed.
As the second one has shown better results, it is this one which has been adopted.

\subsubsection{The coupling matrix}

To carry out the calculation we need first to define what the "local stiffness vectors" for the structure (flanges) and what we 


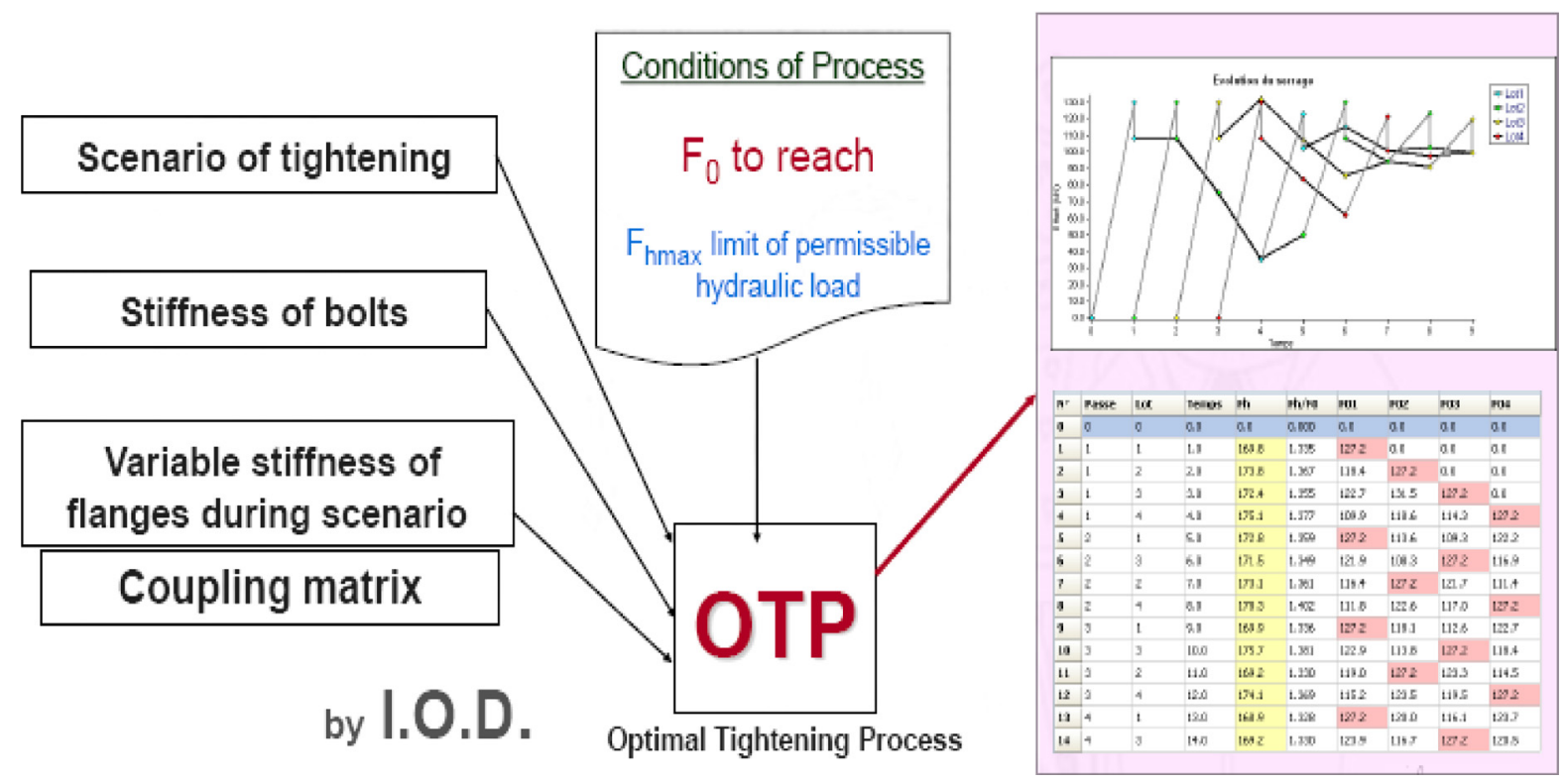

Figure 32. Simplify model explaining the application OTP with bolt tensioner.

call the "coupling matrix" which represents the impact of the tightening of each set of bolts on the sets.

The construction of the "coupling matrix" is first made for displacements $\left[I^{u}\right]$.

It corresponds to the resolution of $X$ different problems at limits; each problem depending on local stiffness at all places where the bolts have already been tightened.

$X$ is the number of sets of bolts which are tightened at same time.

The "coupling matrix" for loads $\left[I^{F}\right]$ is deducted.

\section{Notations}

$n \quad$ Number of the set of bolts currently tightened

$p \quad$ Number of the pass

$j \quad$ Number of the set of bolts for which we are looking at value $k \quad$ Number of the step of tightening

${ }^{k} \delta U_{s j}^{(n), p}$ : Increment of displacement of flange at hole $j$ when bolt $n$ is tightened at pass $p$ (this is the tightening step number $k$ ).

${ }^{k} \delta F_{b j}^{(n), p}$ : Increment of load in the bolt $j$ when bolt $n$ is tightened at pass $p$ (this is the tightening step number $k$ ).

Redundancy between " $k$ " and " $n, p$ " is useful for easier follow-up.

First, we can notice that, as the bolts are all the same there is only one value of stiffness for all of them: $R_{\mathrm{b}}$.

Tightening a set of bolts consists in fact in imposing the tightening length to the concerned bolts.

After tightening this length is the same as the thickness of the compressed flanges at each bolt location of the concerned set.

And then we can deduct the local stiffness of flanges:

$$
{ }^{1} R_{s}^{1}=\frac{{ }^{1} \delta F_{b 1}^{(1), 1}}{{ }^{1} \delta U_{s 1}^{(1), 1}}
$$

This stiffness does depend on $R_{\mathrm{b}}$.

Then the displacements (compressions) at flanges level for the locations of other sets can be deducted.

But local forces at these locations for the first tightening of set 1 are nil.

We so get the coupling factor for displacements:

$$
\frac{{ }^{1} \delta U_{s 1}^{(1), 1}}{{ }^{1} \delta U_{s 1}^{(1), 1}} \rightarrow \frac{{ }^{1} \delta U_{s 2}^{(1), 1}}{{ }^{1} \delta U_{s 1}^{(1), 1}} \quad \frac{{ }^{1} \delta U_{s 3}^{(1), 1}}{{ }^{1} \delta U_{s 1}^{(1), 1}} \quad \frac{{ }^{1} \delta U_{s 4}^{(1), 1}}{{ }^{1} \delta U_{s 1}^{(1), 1}}
$$

Or:

$$
1 \rightarrow{ }^{1} \beta_{2}^{(1), 1}{ }^{1} \beta_{3}^{(1), 1}{ }^{1} \beta_{4}^{(1), 1}
$$

By doing so step by step for the tightening of each set of bolt we finally get the local stiffness vectors and the coupling matrix.

Here after is an example of two circular flanges fastened together by eight bolts.

When tightening the first set of bolts we got:

- The coupling matrix of displacements

$$
I^{u, 1}=\left[\begin{array}{cccc}
1 & { }^{1} \beta_{2}^{(1), 1} & { }^{1} \beta_{3}^{(1), 1} & { }^{1} \beta_{4}^{(1), 1} \\
{ }^{2} \beta_{1}^{(3), 1} & { }^{2} \beta_{2}^{(3), 1} & 1 & { }^{2} \beta_{4}^{(3), 1} \\
{ }^{3} \beta_{1}^{(2), 1} & 1 & { }^{3} \beta_{3}^{(2), 1} & { }^{3} \beta_{4}^{(2), 1} \\
{ }^{4} \beta_{1}^{(4), 1} & { }^{4} \beta_{2}^{(4), 1} & { }^{4} \beta_{3}^{(4), 1} & 1
\end{array}\right] .
$$




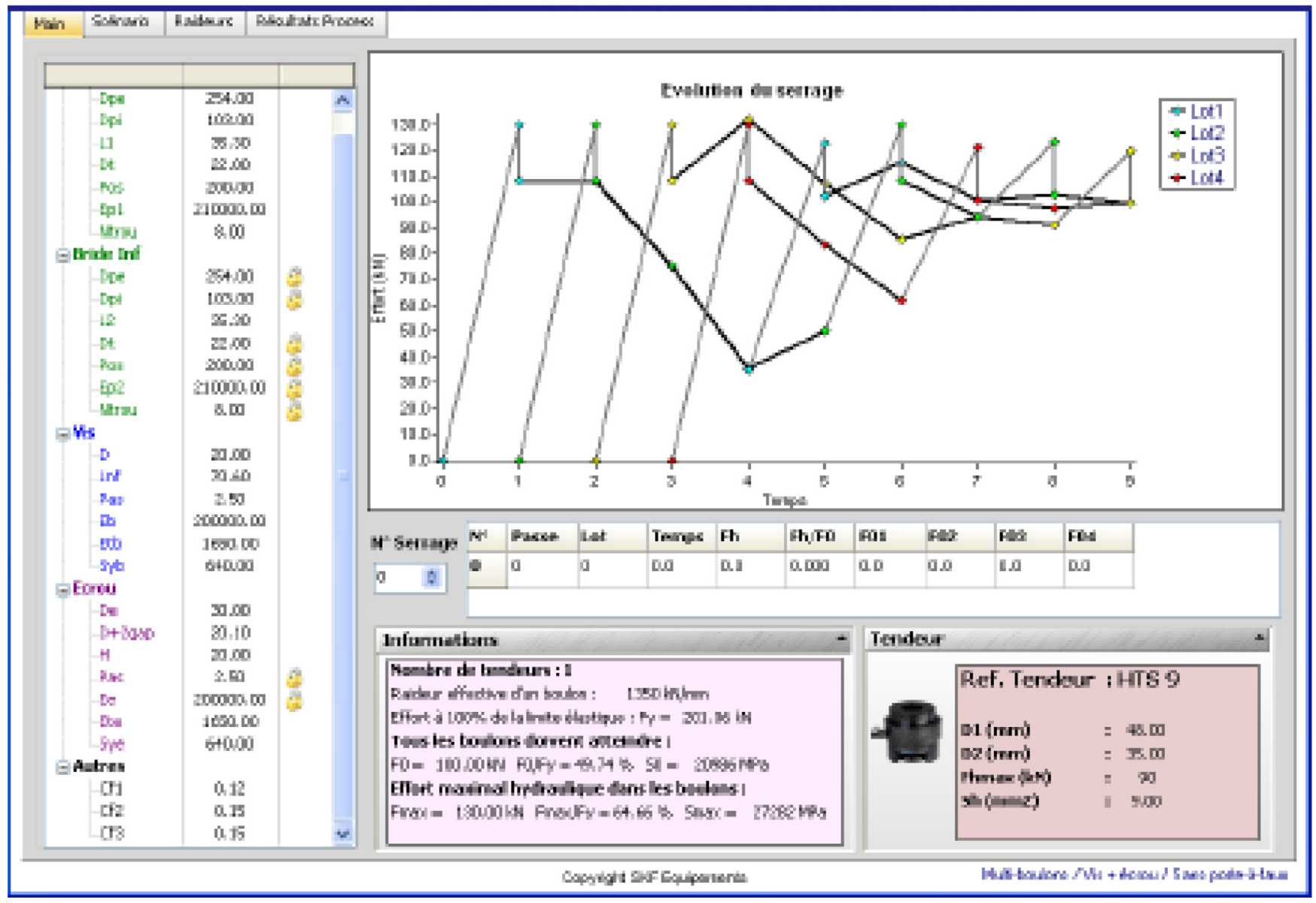

Figure 33. Display screen of the solution given on excel sheet.

- The local stiffness

$$
\left\{\mathrm{R}_{\mathrm{s}}^{1}\right\}=\left\{\begin{array}{c}
{ }^{1} \mathrm{R}_{\mathrm{s}}^{(1), 1} \\
{ }^{2} \mathrm{R}_{\mathrm{s}}^{(3), 1} \\
{ }^{3} \mathrm{R}_{\mathrm{s}}^{(2), 1} \\
{ }^{4} \mathrm{R}_{\mathrm{s}}^{(4), 1}
\end{array}\right\}
$$

- The coupling matrix for load which is deducted

$$
I^{F, l}=\left[\begin{array}{cccc}
1 & 0 & 0 & 0 \\
{ }^{2} \alpha_{1}^{(3), 1} & 0 & 1 & 0 \\
{ }^{3} \alpha_{1}^{(2), 1} & 1 & { }^{3} \alpha_{3}^{(2), 1} & 0 \\
{ }^{4} \alpha_{1}^{(4), 1} & { }^{4} \alpha_{2}^{(4), 1} & { }^{4} \alpha_{3}^{(4), 1} & 1
\end{array}\right]
$$

So, after the local stiffness of the flanges and the coupling matrix are known in addition to the data of stiffness of bolts, the following algorithm can be applied.

\subsubsection{Algorithm of the calculation}

1. Calculation of the initial lengths of each set of bolts for a uniform tightening load $F_{\mathrm{o}}$ applied to all the bolts. At this step, no limitation is fixed.

$$
\left[I^{F, 1}\right]^{T}\left(\begin{array}{c}
{ }^{1} \delta F_{b 1}^{(1), 1} \\
{ }^{2} \delta F_{b 3}^{(3), 1} \\
{ }^{3} \delta F_{b 2}^{(2), 1} \\
{ }^{4} \delta F_{b 4}^{(4), 1}
\end{array}\right)=\left(F_{0}\right)
$$

2. Loop of calculation of the tightening of each set of bolt as long as the calculated initial length does not correspond to the one found at point 1 .

3. Check for each set that the resulting load in the bolts is compatible with the admissible load.

4. Update of control variables: load increment, displacement increment and length compatibility.

5. Deduction of the displacements of the flange at other locations of bolts.

6. Deduction of load of other sets bolts. 

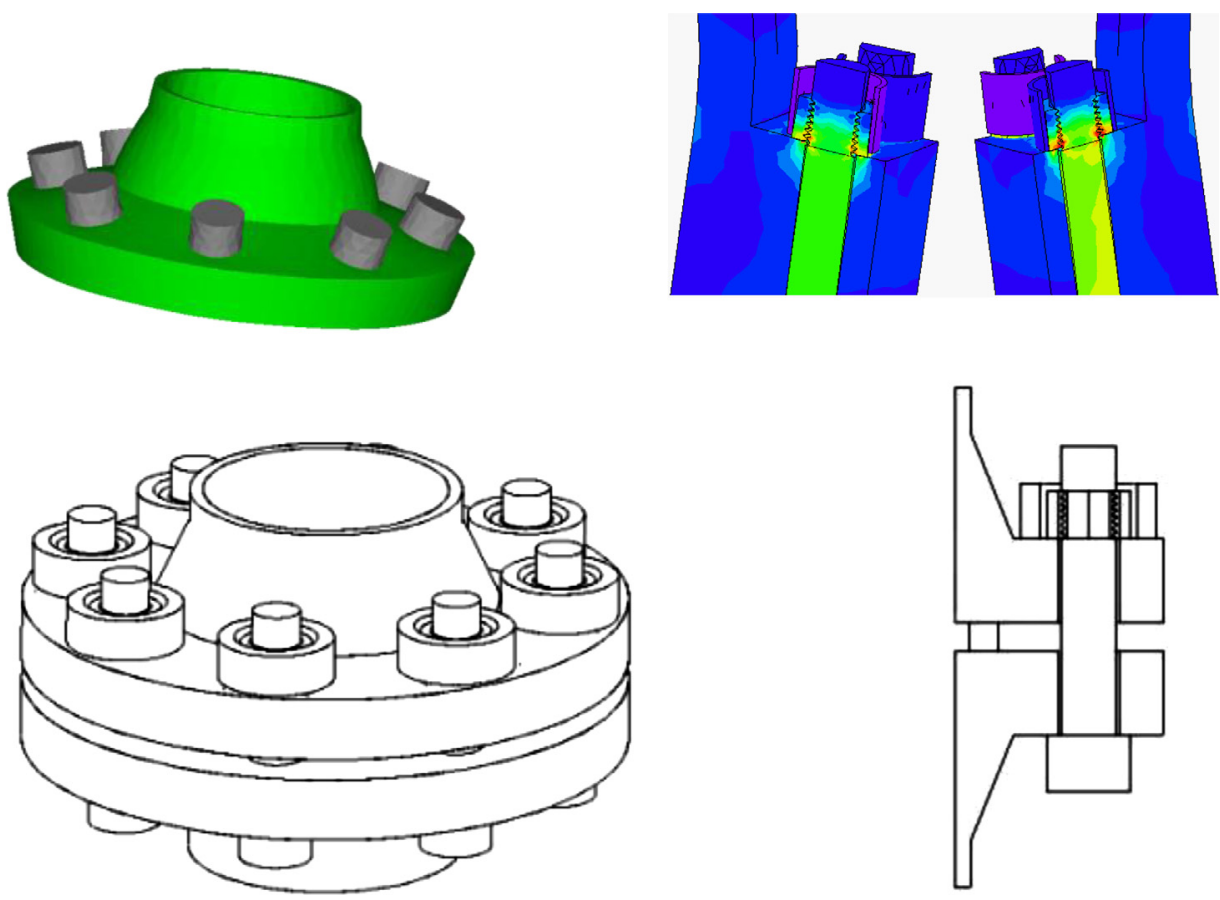

Figure 34. Pictures of the example of a set of two flanges fastened by eight bolts.

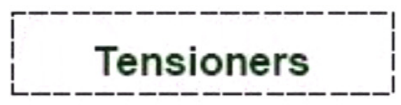

2 hydraulic tensioners
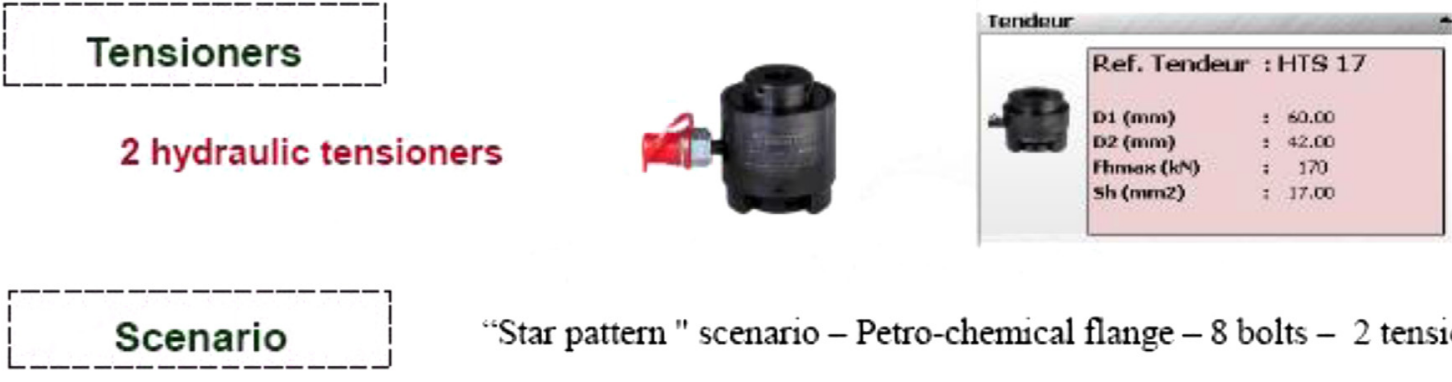

"Star pattern" scenario - Petro-chemical flange -8 bolts -2 tensioners
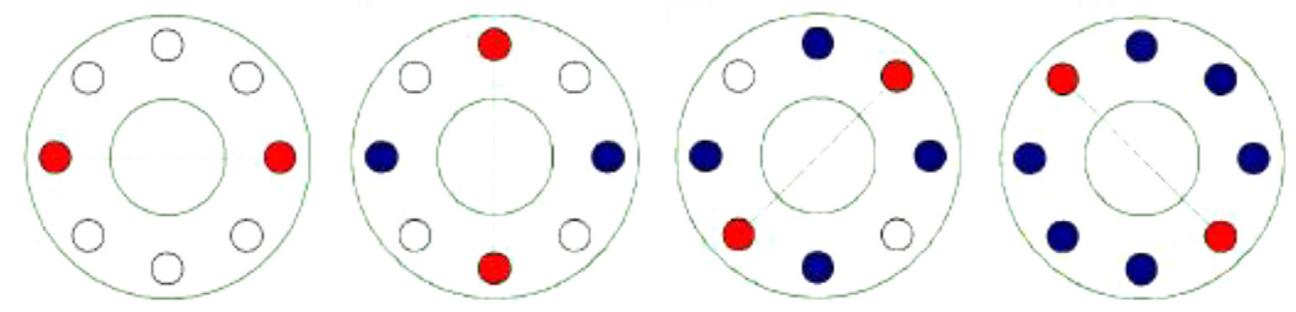

Figure 35. Presentation of the bolt tensioner and the scenario of tightening. model.

All of this can be summarized by the following simplified

\subsubsection{Simplified model behind all of this}

The solution can be displayed on screen, by example on excel sheets (Figures 32 and 33).

\subsubsection{Example of results for an application on a concrete case.}

Two circular flanges assembled with eight bolts (screws and nuts) (Figure 34).

In this example two bolt tensioners are used and the required tightening load is $F_{\mathrm{o}}=100 \mathrm{kN}$ (Figure 35). 


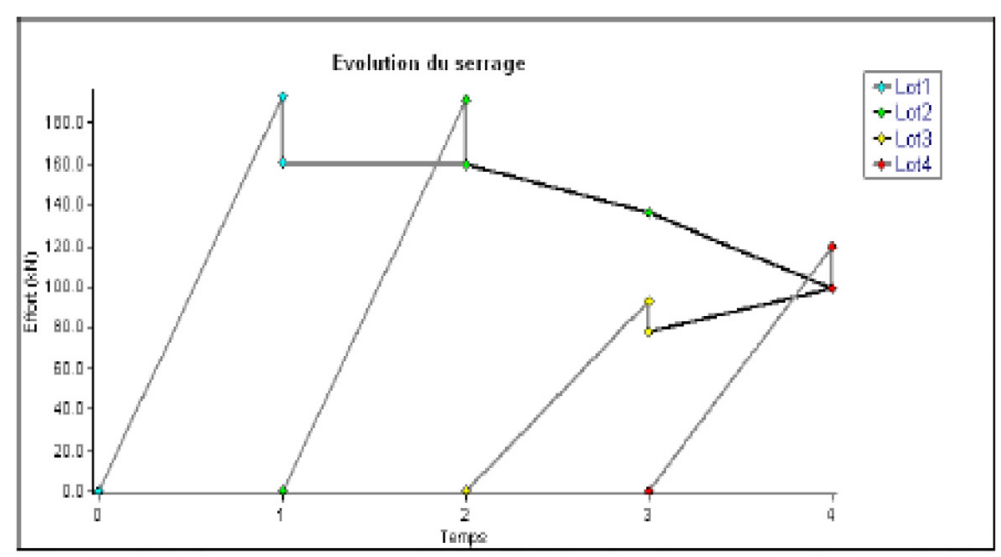

$$
F_{0}=100 \mathrm{kN} \text { to reach }
$$

This one pass process is possible only if NO LIMIT is required for Fh

\begin{tabular}{|c|c|c|c|c|c|c|c|c|c|}
\hline $\mathrm{N}^{\mathrm{a}}$ & Passe & Lot & Temps & fh & $\mathrm{Fh} / \mathrm{FO}$ & Foi & Fu2 & Fôs & FO4 \\
\hline 0 & 0 & 0 & 0.0 & 0.0 & 0.00 & 0.0 & 0.0 & 0.0 & 0.0 \\
\hline 1 & L & 1 & 1.0 & 1980 & 1.200 & 160.8 & 0.0 & 0.0 & 0.0 \\
\hline 2 & t & 2 & 2.0 & 19.6.6 & 1.200 & 159.7 & 159.7 & 0.0 & 0.0 \\
\hline 9 & L & 9 & 3.0 & 98.9 & 1.200 & 1861 & $196: 1$ & 77.7 & 0.0 \\
\hline 4 & $t$ & 4 & 4.0 & 1.9 .3 & 1.200 & 9.5 & 99.5 & 99.4 & 9.5 \\
\hline
\end{tabular}

Figure 36. Results for the case where there is no limitation for $F_{\mathrm{h}}$ load.

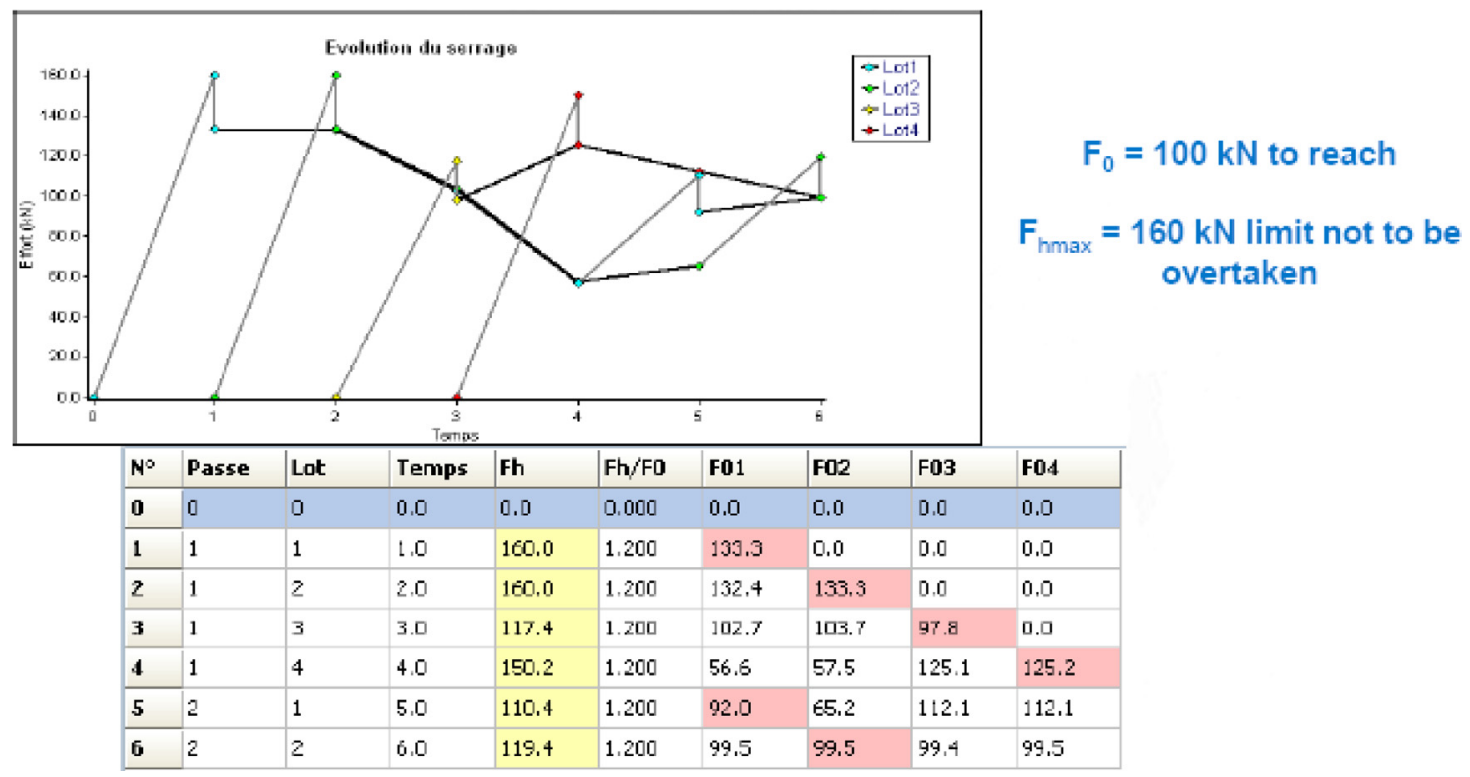

Figure 37. Result for the case where $F_{\mathrm{h}}$ load is limited to $160 \mathrm{kN}$.

Three cases are analyzed in terms of maximum load applied $F_{\mathrm{h}}$ to the bolts at hydraulic tension phase.

- No limitation on maximum load applied to the bolts.

- The maximum load applied to the bolts is limited to $160 \mathrm{kN}$.

- The maximum load applied to the bolts is limited to $120 \mathrm{kN}$.
The results are shown on following the screenshots.

As can be seen and obviously, the lower is the limitation on maximum load $F_{\mathrm{h}}$ on bolts, the higher is the number of necessary passes to reach the required tightening load $F_{\mathrm{o}}$ (Figures 36-38). 


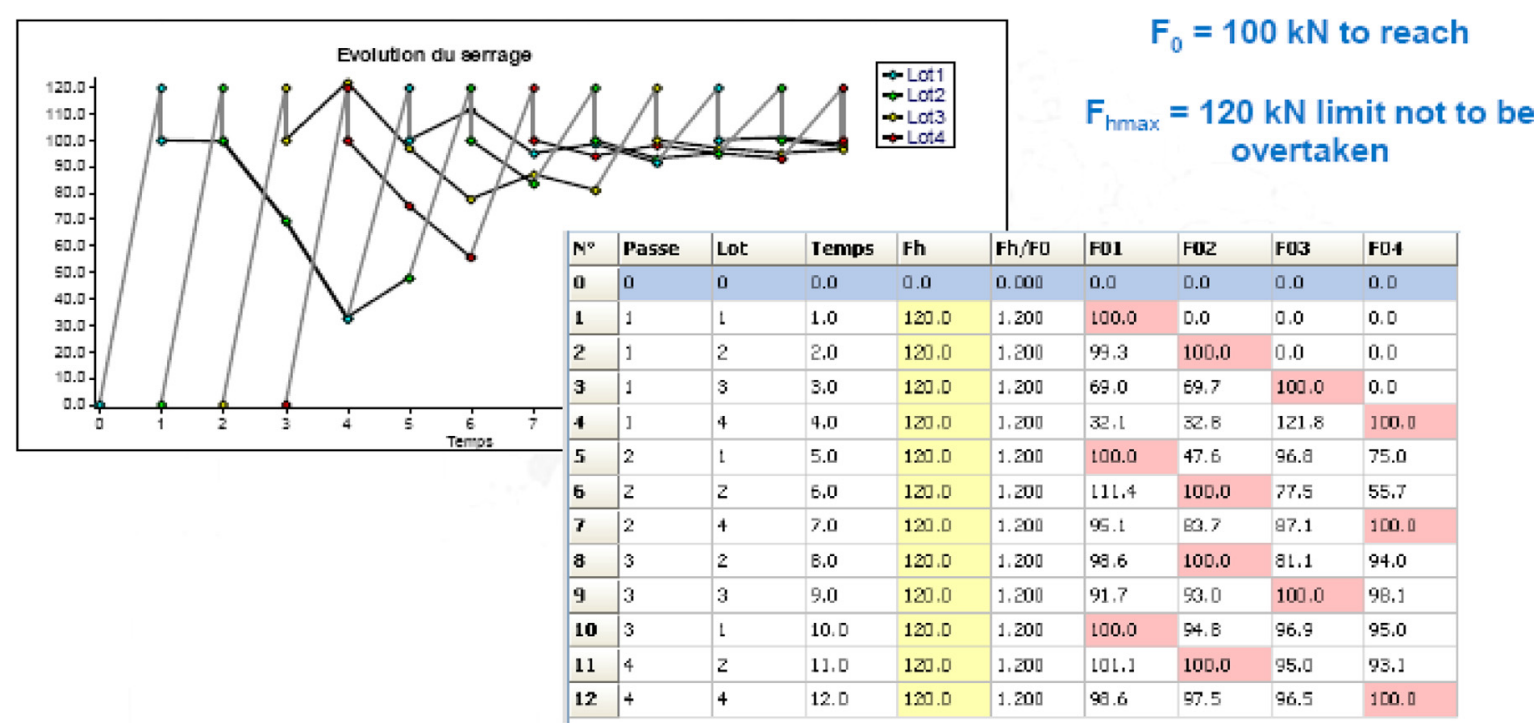

Figure 38. Result for the case where $F_{\mathrm{h}}$ load is limited to $120 \mathrm{kN}$.

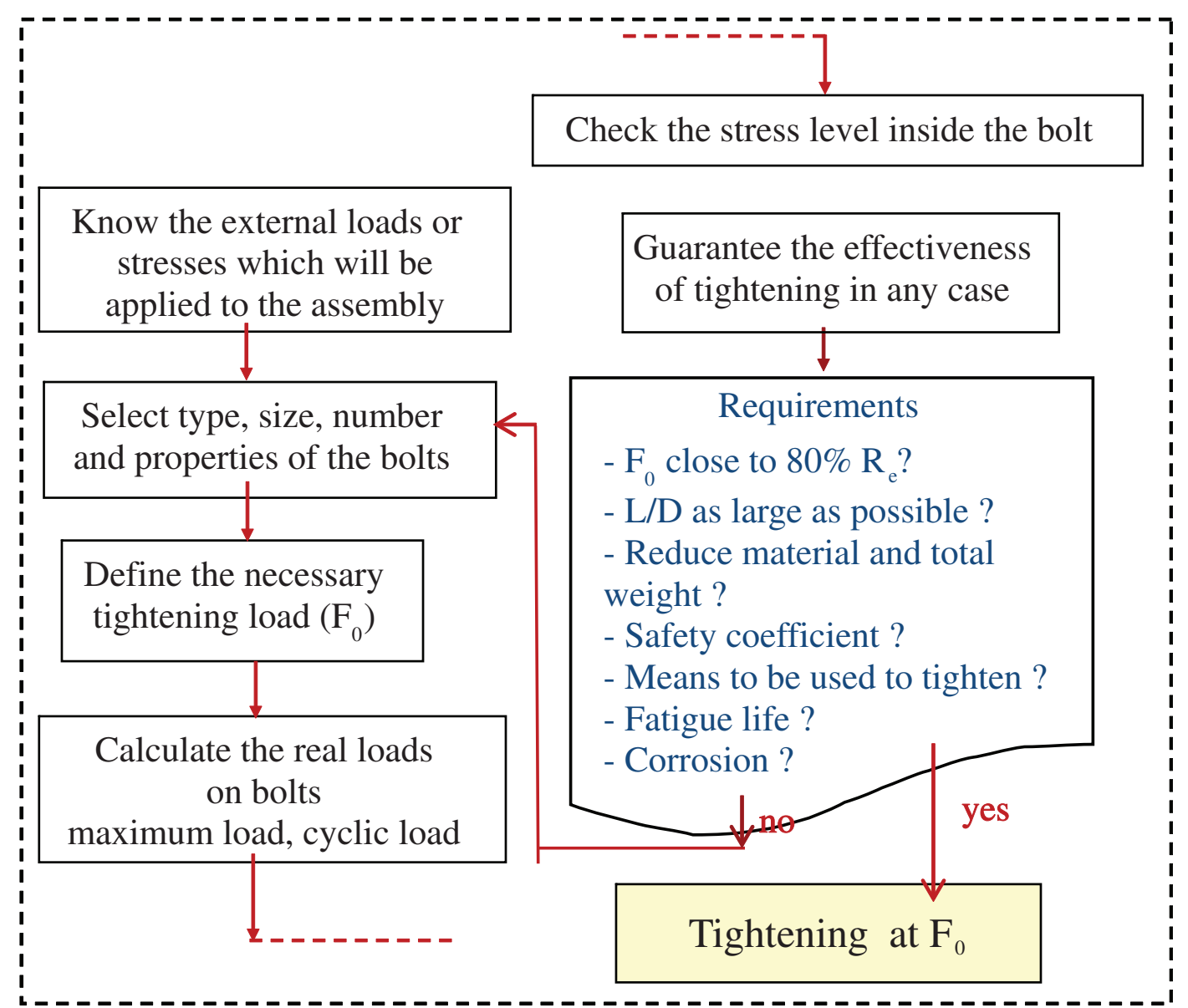

Figure 39. Example of flow chart which could be applied for further development on tools for quicker and more accurate definition of the appropriate tightening load $F_{\mathrm{o}}$ at design stage. 


\section{Conclusion}

We have seen the case of bolted joints already designed, tightened at a load $F_{\mathrm{o}}$ previously defined: single bolt joint or multiple bolt joint having two circular flanges.

For the single bolt applications, the tool allows:

- selecting the most suitable size of bolt tensioner to be used;

- knowing the tightening load $F_{\mathrm{o}}$ which will be obtained when a given tensioning load $F_{\mathrm{h}}$ is applied or knowing the tensioning load $F_{\mathrm{h}}$ to exert by the tensioner in order to obtain the required tightening load $F_{\mathrm{o}}$ taking into account the dimensions of the concerned parts of the assembly and the mechanical properties of the materials.

For the multiple bolt applications, the tool allows:

- selecting the most suitable size of bolt tensioner to be used;

- defining the optimal process taking into account the dimensions of the assembly, the number of tensioners available and the scenario which is selected;

- knowing the hydraulic load $F_{\mathrm{h}}$ to be applied at each pass for each set of bolts to obtain at the end of the process the same tightening load $F_{\mathrm{o}}$ on all the bolts taking into account the dimensions of the concerned parts of the assembly and the mechanical properties of the materials.

A further step concerning bolted joints already designed with tightening load $F_{\mathrm{o}}$ previously defined could be to analyze other shapes of joint members, rectangular or square for example.

The next step would be to deal with new bolted assemblies at early design stage. The challenge would be to define more accurately the most appropriate and optimized tightening load $F_{\mathrm{o}}$ for the application taking into account the static and dynamic loading, the other working conditions and the tightening means and process to be used.

Optimization from the beginning of the design stage (Figure 39).

Acknowledgements. Special thanks to Pr Joseph Zarka and Dr Habib Karaouni for their strong support to write this article.

\section{References}

1. Guillot J. Techniques de 1'Ingénieur Génie Mécanique, France, 1989.

2. SKF. Bolt Tightening Handbook, SKF Montigny le Bretonneux, France, $2001 \& 2009$.

3. Verein Deutscher Ingenieure - VDI 22330, Beuth Vertag Gmbh, Berlin, Germany, 1986-2003.

4. Zarka J, Navidi P. Intelligent optimal design of materials and structures, CADLM, Gif-sur-Yvette, France, 2000.

5. Zarka J, Frelat J, Inglebert G, Kasmai-Navidi P. A new approach in inelastic analysis of structures, CADLM, Gif-sur-Yvette, France, 1990.

6. Bickford JH, Nassar S, Handbook of bolts and bolted Joints, Marcel Dekker, New York, USA, 1998.

Cite this article as: Monville J-M: Optimal tightening process of bolted joints. Int. J. Simul. Multisci. Des. Optim., 2016,7 , A4. 